\title{
Exciton-Polariton Trapping and Potential Landscape Engineering
}

\author{
C. Schneider ${ }^{1}$, K. Winkler ${ }^{1}$, M. D. Fraser ${ }^{2}$, M. Kamp $^{1}$, Y. Yamamoto ${ }^{3,4}$, \\ E. A. Ostrovskaya ${ }^{5}$, and S. Höfling ${ }^{1,6}$ \\ ${ }^{1}$ Technische Physik, Physikalisches Institut and Wilhelm-Conrad-Röntgen-Research Center \\ for Complex Material Systems, University of Würzburg, Am Hubland, D-97074 Würzburg, \\ Germany \\ ${ }^{2}$ Quantum Functional Systems Research Group, RIKEN Center for Emergent Matter \\ Science, 2-1 Hirosawa, Wako-shi, Saitama 351-0198, Japan \\ ${ }^{3}$ ImPACT Project, Japan Science and Technology Agency, Chiyoda-ku, Tokyo 102-0076, \\ Japan \\ ${ }^{4}$ Edward L. Ginzton Laboratory, Stanford University, Stanford, California 94305-4085, USA \\ ${ }^{5}$ Nonlinear Physics Centre, The Australian National University, Canberra ACT 2601, \\ Australia \\ 6 SUPA, School of Physics and Astronomy, University of St Andrews, St Andrews, KY16 \\ 9SS, United Kingdom \\ E-mail: christian.schneiderephysik.uni-wuerzburg.de
}

\begin{abstract}
Exciton-polaritons in semiconductor microcavities have become a model system for the studies of dynamical Bose-Einstein condensation, macroscopic coherence, many-body effects, nonclassical states of light and matter, and possibly quantum phase transitions in a solid state. These low-mass bosonic quasiparticles can condense at comparatively high temperatures up to $300 \mathrm{~K}$, and preserve the fundamental properties of the condensate, such as coherence in space and time domain, even when they are out of equilibrium with the environment. Although the presence of an in-plane confining potential is not strictly necessary in order to observe Bose-Einstein condensation, engineering of the polariton confinement is a key to controlling, shaping, and directing the flow of polaritons. Prototype polaritonbased optoelectronic devices rely on ultrafast photon-like velocities and strong nonlinearities exhibited by polaritons, as well as on their tailored confinement. Nanotechnology provides several pathways to achieving polariton confinement, and the specific features and advantages of different methods are discussed in this review. Being hybrid exciton-photon quasiparticles, polaritons can be trapped via their excitonic as well as photonic component, which leads to a wide choice of highly complementary trapping techniques. Here we highlight the almost free choice of the confinement strengths and trapping geometries that provide powerful means for control and manipulation of the polariton systems both in the semi-classical and quantum regimes. Furthermore, the possibilities to observe effects of the polariton blockade, Mott insulator physics, and population of higher-order energy bands in sophisticated lattice potentials are discussed. Observation of such effects could lead to realization of novel polaritonic non-classical light sources and quantum simulators.
\end{abstract}

PACS numbers: $00.00,20.00,42.10$ 


\section{Introduction}

The term 'polariton' is generally used in solid state physics, when an optical excitation is strongly coupled to a matter excitation. The matter excitation can be provided by a plasmon, a phonon, an electron, or an exciton. This review article exclusively discusses excitonpolaritons in microcavity systems. The excitons, which are bosonic composite quasi-particles consisting of an electron and a hole bound via their Coulomb attraction in a semiconductor, can be confined in quantum well (QW) structures embedded in optical microcavities. If the conditions for strong light-matter coupling are fulfilled, the properties of the bosonic matter excitation and the photon light field inside the microcavity are mixed, and new eigenstates of the coupled system evolve $[1,2]$. Being bosonic quasi-particles, polaritons can in principle condense in a single particle energy state of a finite size [3]. This dynamical condensation of bosons is closely related to the Bose-Einstein condensate (BEC) phase which is usually studied in ultra-cold atomic systems [4]. However, due to the finite lifetime of polaritons even in state-of-the-art microcavities, the thermal equilibrium between the polaritons and their environment is very hard to establish. Nevertheless, the long-range spatial [3,5] and temporal $[6,7,8]$ coherence of polariton condensates has been demonstrated, revealing the characteristic signatures of a BEC [9]. The effective mass of a microcavity polariton is approximately five orders of magnitude smaller than that of a free electron and $8-9$ orders of magnitude smaller than that of an atom. Since the critical temperature for the Bose condensation is inversely proportional to the particle mass [10], polaritons are well suited for the studies of condensation in the temperature range from liquid helium up to room temperature.

Polaritons exist in a semiconductor microcavity environment, hence their properties can be tailored by means of semiconductor lithography and nanotechnology. Manipulation of the polaritonic systems via spatial trapping of excitons and/or photons in real and momentum space can therefore provide access to completely new areas of mesoscopic physics in semiconductors. While photons have to be confined in a cavity to achieve sufficiently long lifetimes to form polaritons, the excitons are usually located in quantum wells to enhance the oscillator strength of the emitters and to reduce possible detrimental effects of surface recombination. Consequently, polaritons formed by the cavity photons and QW excitons are quasi-particles living in a two-dimensional environment of a quantum well-microcavity system. In order to allow for the observation of phase transitions related to Bose-Einstein condensation at finite temperatures, the exciton-polariton system should be of finite size, since otherwise the condensate's long range order would be destroyed by thermal fluctuations [11]. This condition is usually assured by a finite area of optical (or electrical [12]) excitation. Engineering of additional lateral (i.e., in the QW plane) traps in the semiconductor yields the possibility to create sophisticated potential landscapes with the spatial scales of the order of the polariton wavelength $(\sim 1 \mu \mathrm{m})$, and therefore to study interactions and transport of polariton condensates in tailored environments. This possibility, in turn, can be exploited in designing new schemes for highly nonlinear photonic integrated circuits (PICs) and logic elements $[13,14]$. Such PICs promise ultra-high processing speeds and very low power 
consumption, since polaritons can propagate with ultrafast velocities and low decoherence rates. The large polariton nonlinearities inherited from the excitonic component enables manipulation [15], switching and steering of the polariton flow by very weak laser beams [16] and possibly electrical contacts. Additionally, the spin degree of freedom in the polariton system can be actively exploited to add new functionalities to polaritonic logic devices, as discussed in [17].

The possibility to engineer polariton trapping potentials has furthermore triggered the interest in using polaritonic systems to emulate complex many-body phenomena, such as the physics of high-temperature superconductors, graphene, or frustrated spin lattices [18, 19, 20]. Quantum emulators are envisaged as a highly desirable tool for understanding complex manybody properties of novel solid state, chemical, and biological systems, which are otherwise hardly accessible. They rely on the emulation of Hamiltonians via potential landscape engineering in highly controllable quantum systems [21]. Polariton gases in microcavities are considered as promising candidates for solid state quantum emulation, as they fulfill a range of important prerequisites: Namely, they can form bosonic condensates and enter a superfluid phase [22], possess internal (pseudo-spin) degrees of freedom, can be localized by lithographic or optical techniques possibly at the single polariton level, and their interaction constants are tuneable $[23,24]$. One example of progress towards quantum emulations are periodic potential landscapes, where higher-band $\mathrm{p}$ - and d-orbital like condensates mimic the fundamental structure of high temperature superconductors [25, 18], offer the possibility to study Dirac cones [26, 19], and are predicted to implement Bose-Hubbard Hamiltonian physics in semiconductors [27].

Predictions of the quantum blockade regime in the dilute polariton systems have gained significant attention [28, 29, 30]. This effect would allow one to exploit the fascinating properties of polaritons in integrated quantum light sources, sources of entangled and indistinguishable photons [31], and to generate polariton number states in microcavity traps. The latter effect could pave the way for the study of quantum phase transitions and BoseHubbard physics with light, and therefore open new directions in the solid state microcavity research. However, the experimental demonstration of polariton quantum blockade critically relies on the tight trapping of polaritons to enhance polariton-polariton interactions [28], or on the controlled coupling of polariton boxes to facilitate quantum interference [30]. The experimental and technological challenges are significant, and consequently the effect has not been demonstrated yet.

The rapidly expanding research on exciton-polaritons creates an unrelenting demand for elegant and non-destructive methods for trapping polaritons in a microcavity. This review summarizes a number of complementary techniques that have been developed to meet the demand. We discuss the techniques for trapping the excitonic component of the polaritons by manipulating the QWs or by structuring the optical pump, as well as methods for trapping the photonic component by manipulating the microcavity. We review the most prominent experiments enabled by each of the trapping techniques, and discuss the limitations and prospects. 


\section{Theory}

\subsection{Quantum well excitons}

The excitons are localized in the plane of the QWs, however the in-plane exciton wavefunction is usually delocalized over a large number of the crystalline lattice sites due to the electromagnetic screening in the semiconductor with a characteristic Bohr radius of $5-15$ $\mathrm{nm}$ and the binding energy of $4-10 \mathrm{meV}$ for typical III/ $\mathrm{V}$ compounds (except nitrites). Since the physics of quantum well excitons has been studied for several decades, here we will only summarize the most important physical relations and parameters and refer the interested reader to the exhaustive literature (see, e.g. $[1,32,33,34]$ ).

The energy spectrum of a Wannier-Mott exciton in a crystal with a dielectric constant $\varepsilon$ can be found in the effective mass approximation, by solving a Schrödinger's equation for an electron in a hydrogen atom, where the free electron mass, $m_{0}$, has to be replaced by the reciprocal mass $\mu=m_{e} m_{h} /\left(m_{e}+m_{h}\right)$ (here $m_{e}$ and $m_{h}$ are the effective masses of an electron and a hole, respectively), and the dielectric constant $\varepsilon_{0}$ is replaced with $\varepsilon \varepsilon_{0}$.

The Schrödinger equation for the relative electron-hole motion

$$
\left(-\frac{\hbar^{2} \nabla^{2}}{2 \mu}-\frac{e^{2}}{4 \pi \varepsilon \varepsilon_{0} r}\right) \psi(r)=E \psi(r),
$$

where $r$ is the relative distance between the electron and the hole, has a solution in the form of the $1 \mathrm{~s}$ scattering wavefunction

$$
\psi(r)=\frac{1}{\sqrt{\pi a_{B}^{3}}} e^{-r / a_{B}}
$$

with the Bohr radius $a_{B}=4 \pi \varepsilon \varepsilon_{0} \hbar^{2} /\left(\mu e^{2}\right)$ and the corresponding eigenenergy $E=$ $2 \mu e^{4} /\left(8 \pi \hbar \varepsilon \varepsilon_{0}\right)^{2}$.

In quantum confined systems, such as a quantum well, the reduced dimensionality leads to modifications in these quantities, and the Bohr radius, as well as the binding energies of the excitons, critically depend on material parameters. In the presence of confinement, excitonic effects generally are enhanced, resulting, e.g., in a four fold increase of the exciton binding energy in an ideal two-dimensional system. The effects of confinement on excitons are thoroughly investigated in references $[1,32,34,35]$, to list a few.

The oscillator strength of QWs can be expressed in terms of the overlap integral between the electron and hole wavefunctions. Excitonic effects lead to an increase of the oscillator strength compared to band-to-band transitions. The oscillator strength for a transition $f$ can be expressed in the effective mass approximation in terms of the periodic (Bloch) part of the wavefunctions in the valence (v) and conduction band (c) $u_{v, c}$ and the envelope $F_{v, c}$ with the momentum operator $\mathbf{p}$ and the polarization vector $\hat{e}$ :

$$
f=\frac{2}{\mu \hbar \omega}\left|\left\langle u_{v}|\hat{e} \mathbf{p}| u_{c}\right\rangle\right|^{2}\left|\left\langle F_{v} \mid F_{c}\right\rangle\right|^{2},
$$

where $\hbar \omega$ is the energy of the considered transition. 
The oscillator strength is closely related to the optical absorption in a QW as follows:

$$
A=\frac{4 \pi^{2} e^{2} \hbar f}{n m_{0} c L},
$$

where $n$ is the refractive index of the material, $L$ the the well width, and $A$ is the integrated absorption intensity (in units of $\mathrm{eV} / \mathrm{cm}$ ). This quantity can therefore be determined via photon absorption spectroscopy [34].

\subsection{Microcavity Photons}
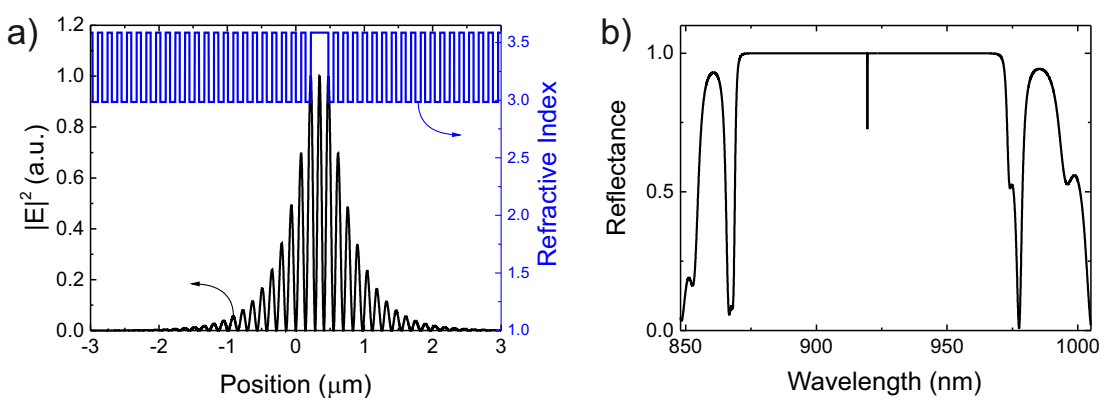

Figure 1. (a) Vertical mode profile in the microcavty: The optical field is strongly enhanced in the central layer, where the QWs are located to maximize light-matter coupling. (b) Calculated reflectivity spectrum of a DBR-microcavity with a strongly pronounced FabryPerot resonance.

There exists a wide range of possibilities to trap photons in semiconductor microcavities in one or more dimensions. Common techniques involve total internal reflection on semiconductor-air interfaces, photonic bandgap tailoring, plasmonic resonances, or distributed Bragg reflection [1]. In the research field of microcavity polaritons, distributed Bragg reflector (DBR) based microcavities are most commonly used, since they can provide near unity reflectivity without additional sample processing and lithography steps. We will briefly summarize their basic properties in this section.

The typical cavity hosting exciton-polaritons is composed of layers with different refractive indices, such as AlAs and $(\mathrm{Al}) \mathrm{GaAs}$. If the thicknesses of the individual layers are chosen to match the Bragg condition, $d=\lambda /(4 n)$, then in such a DBR, interference of transmitted and reflected light allows to achieve extraordinary high reflectivity in a spectral window with a width of about $100 \mathrm{~nm}$ around the Bragg wavelength. In order to form a cavity based on such reflectors, a photonic defect layer has to be included. We will only consider the simplest case here, namely a $\lambda / n$ - thick defect of a high-index material (such as GaAs).

The optical mode profile in such a Fabry-Perot resonator can be calculated by the transfer-matrix method, yielding a strong enhancement of the light intensity inside the cavity defect layer and an oscillating decay inside the DBR mirrors. Details of the method are described in [1]. From this decay, we can define an effective cavity length, which is the sum of the physical cavity length $L_{c}$ and the depth of the light penetration into the mirrors:

$$
L_{\mathrm{eff}}=L_{c}+\frac{\lambda_{c}}{2 n_{c}} \frac{n_{1} n_{2}}{\left|n_{1}-n_{2}\right|}
$$


where $n_{i}$ are the refraction indices of the DBR layers and $n_{c}$ is the refractive index of the cavity layer.

Figure 1(a) depicts the optical field inside of the cavity, as calculated by the plane-wave expansion technique. Additionally, figure 1(b) shows the reflectivity spectrum of the full structure consisting of the microcavity and the surrounding DBRs, which is characterized by a high-reflectivity region (the stopp-band) and the centered resonance dip. The width of this resonance defines the so-called quality factor or Q-factor via $Q=\omega / \delta \omega$, which is directly linked to the lifetime $\tau$ of photons in the cavity $Q=\omega \tau$. In (Al)GaAs DBR based microcavities, Q-factors of several 100000 are available [36, 37] which reflects the high material qualities available by state-of-the-art epitaxy.

As the photon cannot freely propagate through the microcavity, its dispersion relation is strongly modified, and it can be approximated by a parabola for small in-plane momenta, $k_{\|}$, which results in an effective photon mass, $m_{c}$, in a microcavity:

$$
E(k) \approx E_{0}+\frac{\hbar^{2}}{2 m_{c}} k_{\|}^{2},
$$

where $E_{0}=E\left(k_{I I}=0\right)$. The effective mass is defined as $m_{c}=h n_{c} /\left(c L_{c}\right)$ and has a value on the order of $10^{-5} m_{0}$. A detailed derivation of this equation can be found, e.g., in [1, 38].

\subsection{Exciton-polaritons and normal mode coupling}

Implementing a QW with a high oscillator strength in a microcavity with a sufficiently large Q-factor can give rise to a normal mode coupling between the photonic and excitonic resonance. The energy is transferred back and forth from the excitons in the QW to the microcavity photons, which results in a characteristic Rabi oscillation in the time domain accompanied by a normal mode splitting of the polariton branches. A semi-classical treatment of this effect by modeling the system as two damped coupled oscillators is described in $[39,40]$. This coupled oscillator model yields an analytical expression for the normal mode coupling in a straightforward manner. Here, we summarize some important conclusions from this model. Solving the transfer matrix equations yields an expression which connects the frequency of the polariton states with the frequency of the exciton $\omega_{x}$, the cavity resonance $\omega_{c}$, the interaction potential $V$ and the dissipation rates $\gamma_{x, c}$ :

$$
\left(\omega_{c}-\omega-i \gamma_{c}\right)\left(\omega_{x}-\omega-i \gamma_{x}\right)=V^{2} .
$$

This expression yields an equation for $\omega$, which reads

$$
\omega=\frac{\omega_{c}+\omega_{x}-i\left(\gamma_{x}+\gamma_{c}\right)}{2} \pm \sqrt{V^{2}+\frac{1}{4}\left(\omega_{x}-\omega_{c}-i\left(\gamma_{x}-\gamma_{c}\right)\right)^{2}}
$$

The corresponding energies of the upper and lower polariton branch $\hbar \omega$ are plotted as a function of the detuning $\hbar \omega_{c}-\hbar \omega_{x}$ in figure $2(a)$.

The splitting between the upper and lower polariton branches on resonance is the characteristic Rabi splitting $\hbar \Omega$. The Rabi frequency $\Omega$ is then defined as:

$$
\Omega=2 \sqrt{V^{2}-\frac{1}{4}\left(\gamma_{x}-\gamma_{c}\right)^{2}} .
$$


The coupling constant, $V$, is a function of the exciton oscillator strength $f_{n}$, an effective number of QWs in the microcavity, $N_{\mathrm{QW}}$, and the effective cavity length $L_{\mathrm{eff}}$ :

$$
V \approx \sqrt{\frac{2 \Gamma_{0} c N_{\mathrm{QW}}}{n_{c} L_{\mathrm{eff}}}} .
$$

with

$$
\Gamma_{0}=\frac{\pi e^{2}}{n_{c} 4 \pi \varepsilon_{0} m_{e} c} f_{n} .
$$

The hybridization of light and matter leads to a strong modification of the energymomentum dispersion relations. In order to derive the polariton dispersion relations, one needs to introduce the Hopfield coefficients $|X|^{2}$ and $|C|^{2}$ which determine the fraction of light $\left(|C|^{2}\right)$ and matter $\left(|X|^{2}\right)$ for the lower polariton branch:

$$
|X|^{2}=\frac{1}{2}\left[1+\frac{E_{c}-E_{x}}{\sqrt{\hbar^{2} \Omega^{2}+\left(E_{c}-E_{x}\right)^{2}}}\right]
$$

and

$$
|C|^{2}=\frac{1}{2}\left[1-\frac{E_{c}-E_{x}}{\sqrt{\hbar^{2} \Omega^{2}+\left(E_{c}-E_{x}\right)^{2}}}\right]
$$

As a consequence of the parabolic cavity dispersion, the lower branch of the polariton dispersion also acquires a parabolic shape around its energy minimum, and the effective mass of the lower polariton critically depends on the exciton-photon detuning, $\delta=E_{c}-E_{x}$, through the Hopfield coefficients: $m_{L P}^{-1}=|X|^{2} m_{x}^{-1}+|C|^{2} m_{c}^{-1}$ (see. figure 2)

\subsection{Polariton lasing and condensation}

Exciton-polaritons behave as bosons in the low density limit. Due to their light effective mass, they are excellent candidates for studies of bosonic condensation effects and related phenomena at elevated temperatures in semiconductors. However, there are several restrictions:

- Polaritons only live for a short time $(\sim 1-100 \mathrm{ps})$ in a microcavity before they decay radiatively. Hence, they can hardly reach true thermal equilibrium. Therefore, the condensate of exciton-polaritons is often referred to as a dynamic, open-dissipative condensate, or a polariton laser. A comprehensive discussion of the 'polariton laser' vs 'condensate' regime can be found, e.g., in [41].

- At high densities, screening of excitons is dominant, which leads to a bleaching of the oscillator strength and the system enters the (fermionic) plasma phase. This typically happens for particle densities on the order of the Mott transition, i.e $10^{10}-10^{11}$ electronhole pairs per $\mathrm{cm}^{-2}$ [42].

- Excitons are only stable up to a certain temperature which is determined by their binding energy. 

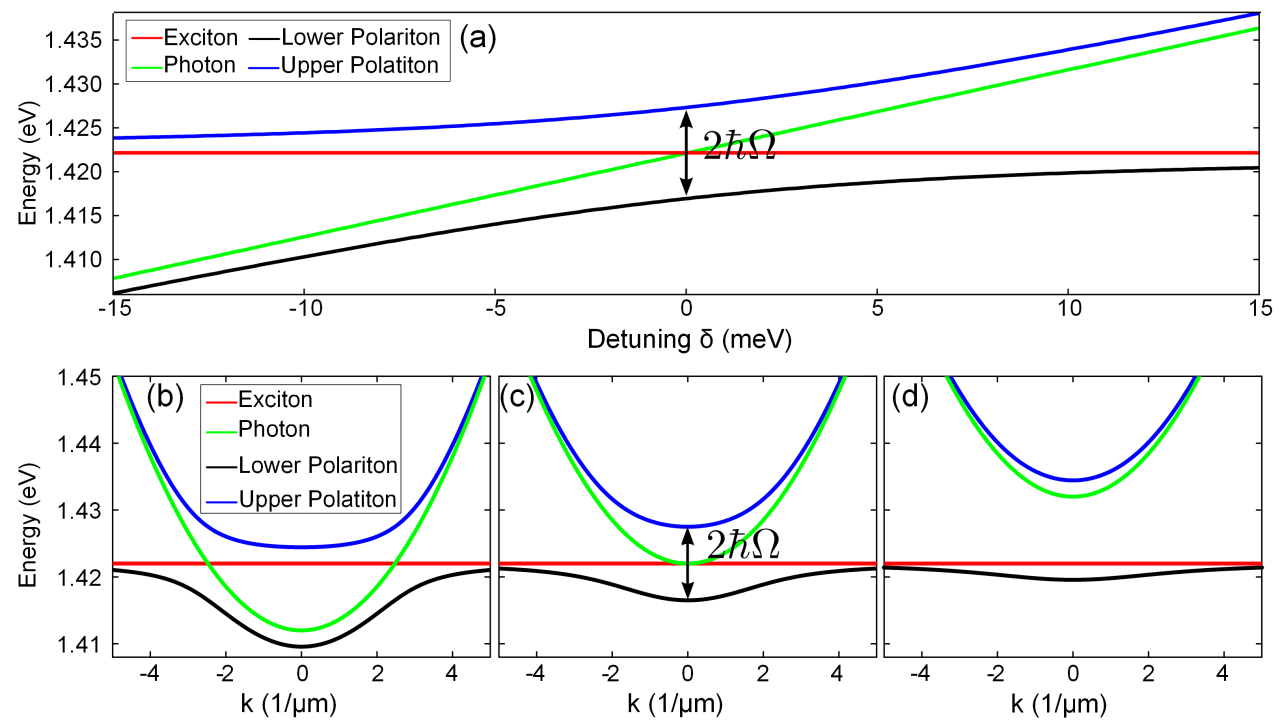

Figure 2. (a) Anticrossing of the lower and upper polariton branch at zero in-plane momentum. Energy-momentum dispersion relations for exciton-polaritons with a large negative detuning of $-\hbar \Omega$ (b), at zero-detuning (c), and with a large positive detuning of $\hbar \Omega(\mathrm{d})$.

The resulting phase diagram of exciton-polaritons has been drawn by Kavokin et al. [1], and is sketched in figure 3 for the case of GaAs QWs in a microcavity.

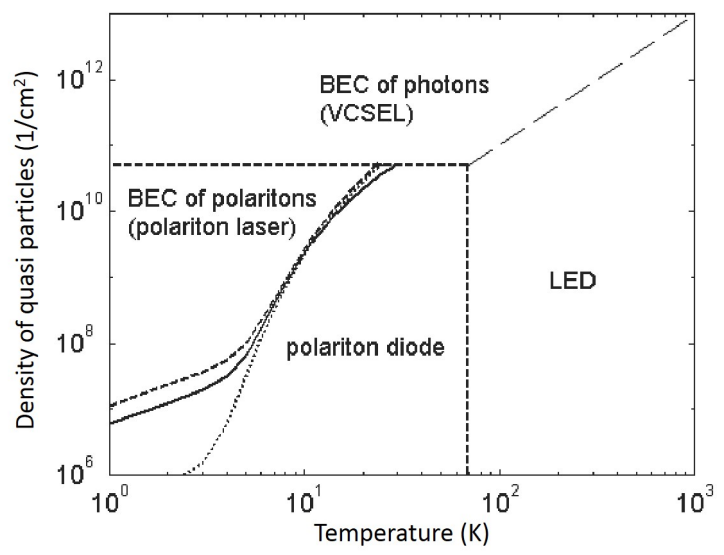

Figure 3. Phase diagram of exciton-polaritons based on GaAs QWs in a microcavity. The image is reproduced in a slightly modified manner from [43].

The planar microcavity exciton-polariton system is inherently two-dimensional (2D), and in accordance with the Hohenberg-Mermin-Wagner theorem, the transition to BEC in 
a uniform system is only possible at zero temperature [11] for 2D as well as 1D geometries. Restriction of the system to a finite size, however, inhibits excitation of density and phase fluctuations permitting the formation of a condensate or quasi-condensate phase with a macroscopic coherence length. This is true for a polariton system with a finite excitation area, both in one and two dimensions $[44,45]$. In most cases, the coherence length of the polariton condensate is thus finite, and ranges on the order of the optical pumping spot size [3]. At macroscopic lengths, and in the $2 \mathrm{D}$ case, it is generally assumed that the system enters the Berezinskii-Kosterlitz-Thouless (BKT) phase, where vortex-antivortex pairs are formed, and the spatial correlation function decays according to a power law [46, 47]. Although the correlation function measurements in exciton-polariton system are suggestive of the BKT phase [48] more conclusive detection of spontaneously formed vortex pairs is required to confirm this regime.

The need to confine polaritons goes far beyond the unambiguous observation of condensation effects. As described in the introduction, the engineered confinement of excitonpolaritons paves the way for creating functional polaritonic circuits and quantum simulators, as well as fundamental studies of polariton condensation in complex potential landscapes.

\subsection{Polaritons in potential landscapes}

Since exciton-polaritons are composed of light and matter, both the photonic and the excitonic part of the quasiparticle can be subject to confinement. Theoretical description of the excitonpolaritons in the engineered potentials below condensation threshold generally relies on calculating the eigenstates of photons and excitons under respective confinement conditions and then using the exciton-photon interaction Hamiltonian to derive the corresponding polariton dispersion. This approach was successfully used, e.g., to describe discrete polariton states appearing in a zero-dimensional 'mesa' trap [49]. Indeed, as will be discussed below in section 3.2.5, such a trap provides a purely photonic confinement of a polariton, and therefore it is sufficient to solve a Maxwell equation for the photon field, $\mathscr{E}(\rho)$ with the boundary conditions given by the contour of the mesa, and assuming that at a position $\rho$ within the trap the electromagnetic modes are locally equivalent to the planar cavity modes: $\mathscr{E}(r)=\mathscr{E}(\rho) \exp \left(i k_{z}(\rho) z\right)$, where $k_{z}(\rho)$ is a suitably modified propagation constant. Introducing the field operators of cavity photons, $\hat{\phi}$, and excitons, $\hat{\chi}$, the second-quantized Hamiltonian for the two coupled oscillators model (7) [38] can be written as:

$$
\begin{aligned}
& \hat{H}=\sum_{\{n\}}\left(\hbar \omega_{c\{n\}} \hat{\phi}_{\{n\}}^{\dagger} \hat{\phi}_{\{n\}}+\hbar \omega_{x\{n\}} \hat{\chi}_{\{n\}}^{\dagger} \hat{\chi}_{\{n\}}\right)+ \\
& \sum_{\{n\},\left\{n^{\prime}\right\}}\left(\hbar \Omega_{\{n\},\left\{n^{\prime}\right\}} \hat{\phi}_{\{n\}}^{\dagger} \hat{\chi}_{\left\{n^{\prime}\right\}}+\text { h.c. }\right),
\end{aligned}
$$

where summation over all quantum numbers of the bound states is assumed. Here $\hbar \omega_{c, x}$ are the eigenenergies of the photon and exciton modes, and $\Omega_{\{n\},\left\{n^{\prime}\right\}}$ is defined through the Rabi splitting in the planar region, $\Omega$, and the overlap integrals between the corresponding exciton and photon modes [49]. In the absence of excitonic confinement, exciton modes are those of a free particle. 
Similarly, using the linear exciton-photon coupling Hamiltonian (7) and (13), it was shown that the structure of the energy bands imposed either on the cavity photon mode (or the exciton mode) by a periodic potential (e.g., periodic array of mesas) translates into the bandgap structure of the polariton spectrum $[50,51]$. For the case when only photonic modes are affected by the periodic potential, the energy bands of the lower-polariton (LP) state are determined as:

$$
E_{n}^{L P}=\frac{1}{2}\left[E_{x}+E_{c n}-\sqrt{\hbar^{2} \Omega^{2}+\left(E_{c n}-E_{x}\right)^{2}}\right],
$$

where $E_{c n}=\hbar \omega_{c}$ are the photonic Bloch bands, and $E_{x}=\hbar \omega_{x}$. Alternatively and equivalently, the LP band structure can be calculated directly by solving the eigenvalue problem for the polariton Bloch states $u_{\mathbf{k}}(\boldsymbol{r})=u_{\mathbf{k}}(\boldsymbol{r}+\mathbf{a})$ in an effective polaritonic in-plane potential $V(\boldsymbol{r})=V(\boldsymbol{r}+\mathbf{a})$ :

$$
\left[\frac{\hbar^{2}}{2 m_{L P}}(-i \nabla+\mathbf{k})^{2}+V(\boldsymbol{r})\right] u_{(n, \mathbf{k})}(\boldsymbol{r})=E_{n}(\mathbf{k}) u_{(n, \mathbf{k})}(\mathbf{r})
$$

where $n$ is the band index and $m_{L P}$ the effective polariton mass in the planar region. A typical structure of LP energy bands $E_{L P}(\mathbf{k})$ is plotted in figure 4 for a square lattice with $a=3 \mu \mathrm{m}$, and the comparison with the low density polariton emission spectrum (see section 3.2.5, figure 16) demonstrates a good agreement. Furthermore, provided the effective polariton potential is deduced with sufficient accuracy, a fractal band-gap spectrum in a Fibbonacci lattice has also shown to be well reproduced by this approach [52]. For a considerable polariton density above the condensation threshold, the energy spectrum is significantly affected by the polaritonpolariton interaction leading to screening of the band-gap structure [53] and formation of localized gap states $[54,55,51,56]$.

Above the condensation threshold, the linear coupled oscillator model no longer holds. With the exception of a very strong confinement in zero-dimensional structures, static and dynamic features of the exciton-polaritons condensates in trapping potentials can be well captured by the mean-field description. The mean-field models are derived under the assumption that a significant population is present in each of the intracavity modes, as well as near the energy minimum of the LP dispersion, such that bosonic stimulated scattering into the condensed state is notable.

In the case of coherent, resonant excitation of the condensed state, such as the OPO regime [57], the modified Gross-Pitaevskii equation for the cavity photon and QW exciton mode amplitudes can be written in the form:

$$
\begin{aligned}
& i \partial_{t} \Psi_{c}=\left(\omega_{c}^{0}-\frac{\hbar^{2}}{2 m_{c}} \nabla^{2}-i \gamma_{c}\right) \Psi_{c}+\frac{\Omega}{2} \Psi_{x}+\alpha \mathscr{E}_{p} \\
& i \partial_{t} \Psi_{x}=\frac{\Omega}{2} \Psi_{c}+\left(\omega_{x}^{0}+V(\mathbf{r}, t)-i \gamma_{x}\right) \Psi_{x}+G\left|\Psi_{x}\right|^{2} \Psi_{x} .
\end{aligned}
$$

where $\mathscr{E}_{p}(\mathbf{r}, t)=\mathscr{E}_{0} \exp (i \mathbf{k r}) \exp \left(-i \omega_{p} t\right)$ is the optical pump field, $\alpha$ is the response coefficient, $G$ is the exciton-exciton interaction strength, and the exciton and photon modes are characterized by their minimum energies $\hbar \omega_{c, x}^{0}$. The model in this form was successfully used, e.g., in [53] to describe dynamics of condensation in a periodic potential $V(\mathbf{r}, t)$ imposed predominantly onto excitonic component of polaritons by surface acoustic wave modulation. 
Note that the effective mass of the excitons is much larger than that of cavity photons, so that their kinetic energy is ignored in Eq. (16).

The model equation (16), with the external potential term introduced into the equation for the cavity photon amplitude, could also serve to describe resonant excitation and nonlinear dynamics of exciton-polaritons in photonics potentials, e.g., those provided by tapered microcavities [16]. It is important to note, however, that in order to describe realistic experimental situations, the mean-field models often need to be augmented to account for non-radiative energy relaxation of excitons. This is usually done by introducing a phenomenological damping term into a modified Gross-Pitaevskii equation [58] in the spirit of thermal relaxation models for atomic condensates [59]. Recently, a stochastic Gross-Pitaevskii equation for the polariton order parameter $\Psi_{L P}$ was derived from the full microscopic theory, under the assumption of coherent pumping and phonon-assistend energy relaxation [60], and shown to be successful in describing thermal relaxation of a polariton condensate in an imposed potential landscape.

Finally, as will be discussed in detail in the subsequent section 3.1.5, incoherent, faroff resonant excitation of a polariton condensate provides a unique opportunity to create reconfigurable traps for polaritons induced by an optical pump via an excitonic reservoir. The model for the reservoir-coupled condensate is discussed, e.g., in [61]. In a more general form, this open-dissipative Gross-Pitaevskii equation (or modified complex Ginzburg-Landau equation), takes the form:

$$
\begin{aligned}
i \hbar \frac{\partial \Psi_{L P}}{\partial t} & =\left[-\frac{\hbar^{2}}{2 m_{L P}} \nabla_{\perp}^{2}+g_{c}\left|\Psi_{L P}\right|^{2}+g_{R} n_{R}(\mathbf{r}, t)+i \frac{\hbar}{2}\left(R n_{R}(\mathbf{r}, t)-\gamma\right)\right] \Psi_{L P}, \\
\frac{\partial n_{R}}{\partial t} \quad & =-\left(\gamma_{x}+R\left|\Psi_{L P}\right|^{2}\right) n_{R}(\mathbf{r}, t)+P(\mathbf{r}) .
\end{aligned}
$$

Here $\Psi_{L P}$ is the condensate wavefunction, $n_{R}$ is the reservoir density, and $P(\vec{r})$ is the spatially modulated optical pumping rate. The critical parameters defining the condensate dynamics are the loss rates of the polaritons $\gamma$ and reservoir excitons $\gamma_{x}$, the stimulated scattering rate $R$, and the strengths of polariton-polariton, $g_{c}$, and polariton-reservoir exciton, $g_{R}$, interactions. Even without an engineered confining potential, the polaritons can be spatially localized in the vicinity of a single pump spot due to the gain-induced self-trapping effect, which is generic for open-dissipative systems [62, 63, 64]. Combination of several pump spots or pumping with structured light beams creates an effective polariton trap due to a strong repulsive potential induced by the reservoir density distribution $n_{R}(\mathbf{r})$. A striking effect of this all-optical trapping scheme is shown in figure 4(b), where the condensate is confined in an effective 1D harmonic potential created by two intense pump spots. The model (17) with various modifications accounting for the adiabatic evolution of the reservoir (see, e.g., [65]) phenomenological energy relaxation, and reservoir diffusion, is successfully used to describe experimental observations of all-optically trapped polariton condensates [66, 67, 68, 69]].

Theoretical and numerical studies of the model (17) have also resulted in a number of predictions concerning the behaviour of far off-resonantly excited polariton condensates in the presence of an engineered trapping potential. For example, trap-induced deformations of the polariton density $[65,63]$, instabilities promoting vortex formation $[65,70]$, and detailed 
structure of excited states in harmonic potentials [71] were described theoretically, but still await to be systematically tested in experiments.

Finally, it has been predicted that in the presence of spatial confinement, polariton interactions can be notably enhanced [28]. The nonlinear coefficient characterising the polariton interaction in the presence of optical confinement within a cylindrical area of radius $r_{0}$ is calculated as a function of the structure parameters in [28]. The interaction energy reads: $\hbar \omega_{n l}=2.67 \hbar \kappa /\left(2 r_{0}\right)^{2}$, where $\kappa$ quantifies the polariton blueshift without lateral confinement, and ranges in the order of $\hbar \kappa=1.5 \times 10^{-} 2(\mu \mathrm{m})^{2} \mathrm{meV}$. If the value for $\omega_{n l}$ exceeds the polariton linewidth, only one polariton can be injected into the system by a resonant laser, and the regime of polariton blockade is reached.
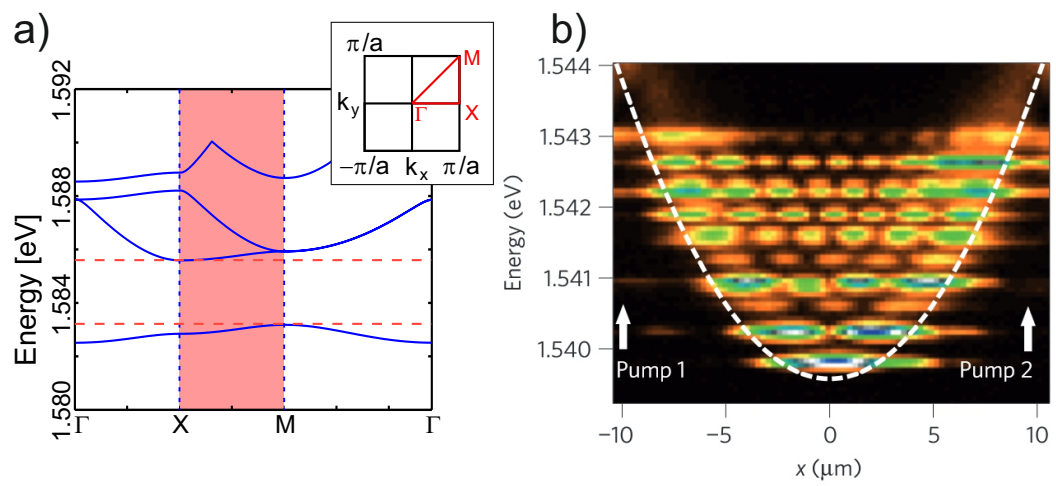

Figure 4. (a) The first four energy bands for a square lattice array of polariton traps in the reduced Brillouin Zone (BZ) representation. Dashed lines indicate the position of the complete band gap. Inset: The first irreducible BZ. (b) Harmonic energy spectrum of a polariton condensate trapped between two laser spots. This figure is reproduced with permission from [66].

\section{Experimental approaches to polariton confinement}

In the following, we will discuss various techniques employed to spatially confine polaritons and highlight the advantages and limitations of each technique. We also summarize important experiments made possible by the techniques under consideration. We will first discuss methods for confining polaritons via their excitonic part. In the second half of this section we will review commonly used approaches to trapping the polaritons via their photonics component.

\subsection{Trapping polaritons via the excitonic part}

For all techniques employed to localize microcavity polaritons via their excitonic part, it is of crucial importance to retain a high quantum efficiency, prevent surface recombination, and preserve a large exciton oscillator strength. These design rules naturally exclude some methods, such as defining small quantum dots (QDs) via lithography and dry etching of a QW, and set intrinsic limits to others (e.g., application of large local electric fields). 

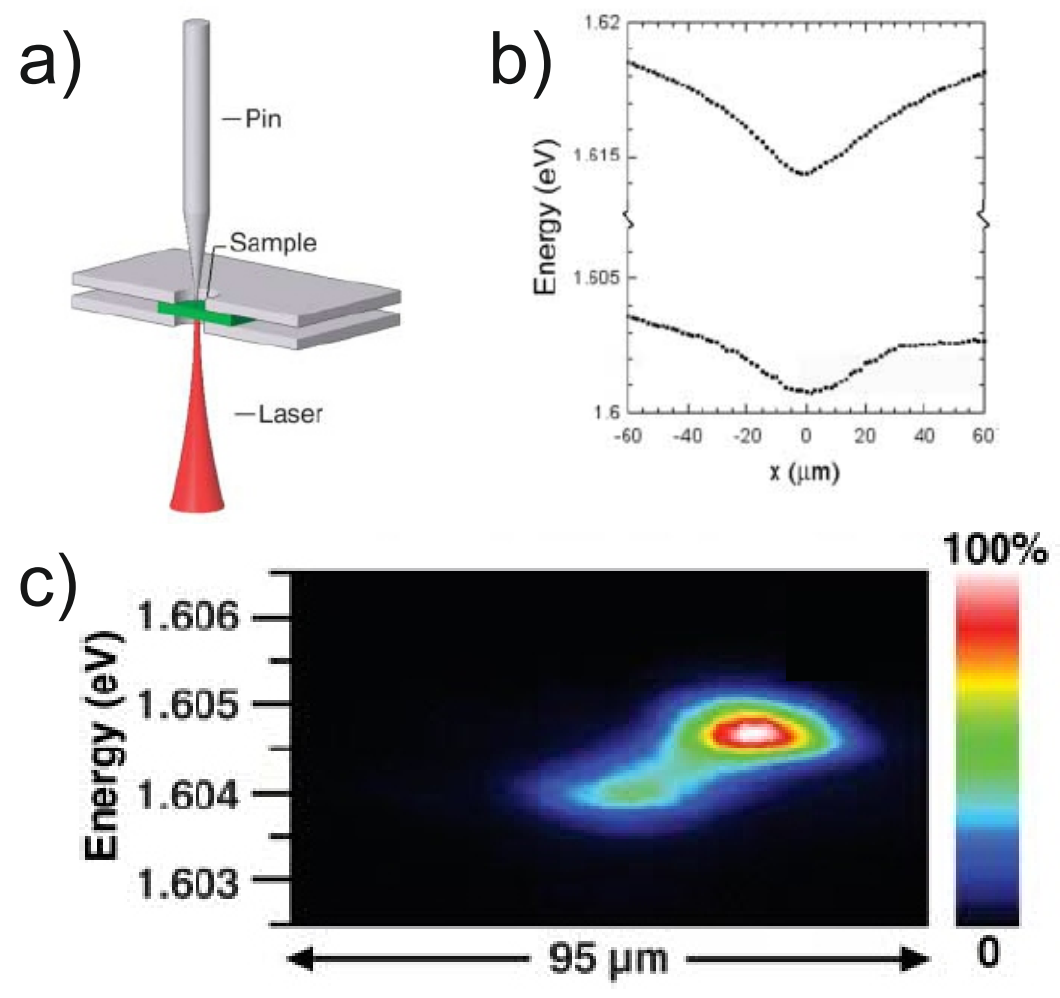

Figure 5. (a) Schematic drawing of the setup to apply local strain to a sample via a pin. (b) Potential profile on polaritons trapped in such a strained environment. (c) Flow of a polariton condensate from the excitation spot (on the right) into the potential minimum created by local strain. The figure is reproduced with permission from [5].

3.1.1. Application of local strain Since the bandgap of semiconductor crystals critically depends on the distance between two nearest atomic neighbors, externally applied crystal strain can be employed to locally tune the exciton wavelength. One possible scheme exploiting this effect to create a trapping potential for polaritons is presented in figure 5(a) [5]. The sample with a partly removed substrate is held in the cryostat, and a sharp metal tip with a radius of $50 \mu \mathrm{m}$ locally applies compressive strain on the reverse side. In this manner, a local parabolic potential minimum for QW excitons on the order of the width of the tip can be created. The local redshift of the exciton energy at the position of the tip is also reflected in the energy minimum for exciton polaritons which is shown in figure 5(b). The depth of this potential can reach several tens of $\operatorname{meV}[5,72,73,74]$, clearly exceeding the thermal energy at liquid helium and even nitrogen temperatures. The implementation of this technique led to the first successful demonstration of Bose-Einstein condensation of exciton-polaritons in a GaAs based microcavity under strictly non-resonant and non-local pumping, taking advantage of the diffusion of polaritons into the trap. This effect can be seen in the real space image in figure 5(c). The polaritons are generated by a non-resonant pump laser on the right side of the trap. The emission shown in figure 5(c) is a consequence of polaritons flowing into the potential minimum separated by $\sim 20$ microns from the excitation. The flexibility to adjust 
the strain reversibly, and hence control the potential shape and depth is another advantage of this technique. However, due to the finite size of the mechanical pin, more complex potential landscapes beyond a single trap seem difficult to realize. The lateral trap size on the $10-100$ $\mu m$ scale makes this technique, at least in this implementation, unsuitable for the observation of size quantization effects or blockade effects on the single polariton level.

3.1.2. Surface acoustic waves A somewhat related technique, which also exploits a modification of the local strain environment to manipulate the polariton potential landscape, is the application of surface acoustic waves (SAWs) to the polariton system. The field can, for instance, be electrically stimulated by an interdigitated transducer. The sound waves of the acoustic field then propagate along the sample, as sketched in figure 6(a) for a two dimensional square configuration. Strictly speaking, and similarly to the local static strain technique, the acoustic phonon field has a direct impact on both the excitonic and the photonic part of the polariton:

- As discussed above, the strain pulse locally affects the energy of the QW-exciton via the stress depending bandgap, or more precisely, via the deformation potential interaction energy. This effect is the stronger one of the two.

- Both the physical cavity length and the refractive index of the cavity material are locally modified in the presence of the acoustic field, which leads to a change of the local resonance condition and hence to optical confinement.

The depth of the excitonic confinement exceeds the photonic modulation by more than a factor of two, yet both effects on their own are sufficiently strong to provide effective polariton localization [75]. Figure 6(b) depicts the influence of the SAWs on the bandstructure of the lower polariton branch. As a result of the generation of a two-dimensional polariton superlattice, a strong modification of the polariton dispersion is evidenced from the calculations, as depicted in figure 6(b) [55]. In such a potential landscape, the condensation of polaritons close to the M-point of the BZ in the lattice bandgap was observed, where the effective mass of the polaritons acquires a negative sign.

3.1.3. Proton implantation induced intermixing Local interdiffusion of compound QWs with the barrier material is a promising technique to generate large excitonic trapping potentials with lateral extensions below $10 \mu \mathrm{m}$. Intermixing techniques have been widely studied in semiconductor laser research [76], and offer a convenient way to tailor QW properties post-growth. However, this comes at the expense of a loss in flexibility, since intermixing naturally is an irreversible process.

One possible scheme to locally tune a QW via rapid thermal annealing without damaging its properties can be facilitated by masking the QW-cavity wafer with a material of very low heat transmittance. Polariton traps can be created at these positions after the annealing process, as the annealing procedure leads to a local redshift of the QW emission. SrF has been identified to be a good candidate for this application [76]. Underneath the masked areas the GaAs QWs (for instance embedded in AlAs barriers) remain widely unaffected by the 
a)

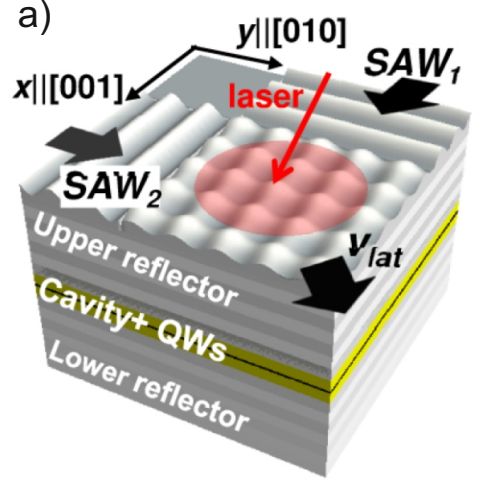

b)

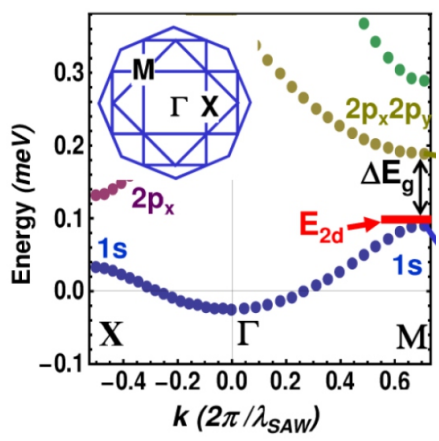

Figure 6. (a) Schematics of two propagating SAWs, forming a square lattice potential on a polariton sample. (b) Calculated bandstructure of the lower polaritons arising in such a periodic potential. The figure is reproduced from [55]. Copyright (2013) by The American Physical Society.

annealing process if moderate temperatures below $900^{\circ} \mathrm{C}$ and reasonable annealing times are applied. In the surrounding areas, which could for instance be covered by a heat transmitting dielectric or a diffusion enhancing material, such as $\mathrm{SiO}_{2}$, the QW emission energy can be blue-shifted via QW-barrier intermixing.

An alternative technique to induce a local intermixing between the QW material and its barrier employs the deliberate disordering and annealing of the semiconductor heterostructure interfaces by high energy ion implantation [77]. Depending on the atomic species being implanted and the dose (ions $/ \mathrm{cm}^{2}$ ), a rapid thermal anneal can recover the induced damage of the ion beam to a high degree. Protons are the natural choice of ion for this technique, as hydrogen is a common, largely inactive interstitial atom incorporated during growth, and will have a negligible effect on the post-processed material quality.

Despite the thickness of the top DBR layer, the ion energy can be tuned specifically such that the protons deposit the bulk of their energy in the QW-embedded cavity region, thus creating the highest density of vacancies. Figure 7(a) shows the simulated ion and vacancy profile in a typical GaAs microcavity structure with $24 / 27$ mirror pairs on the top/bottom of the cavity. For the chosen energy of $440 \mathrm{keV}$, the ion and vacancy concentration is largely localized to the cavity region.

The applicability of this technique is strongly supported by previous studies [78] of proton implantation-induced intermixing on bare GaAs/AlGaAs QWs which show energy shifts in the 100's of meV range, and specifically, that at smaller doses, the luminescence intensity and linewidth can be recovered close to original values but still with $10 \mathrm{~s}$ of meV shifts, sufficient for a tight and deep trapping of exciton-polaritons in microcavities.

The shift of the lower polariton (LP) state will strongly depend on the initial cavityexciton detuning. Given a blue-detuned LP state [figure 7(b)], as nominal exciton energy $E_{X}^{0}$ is shifted to the blue (and correspondingly the LP energy) with increasing dose, the lower polariton detuning will shift to the red [figure 7(c)]. As far red-detuned polaritons are quite insensitive to the exciton energy, the LP mode will only shift to a maximum energy roughly 
(a)

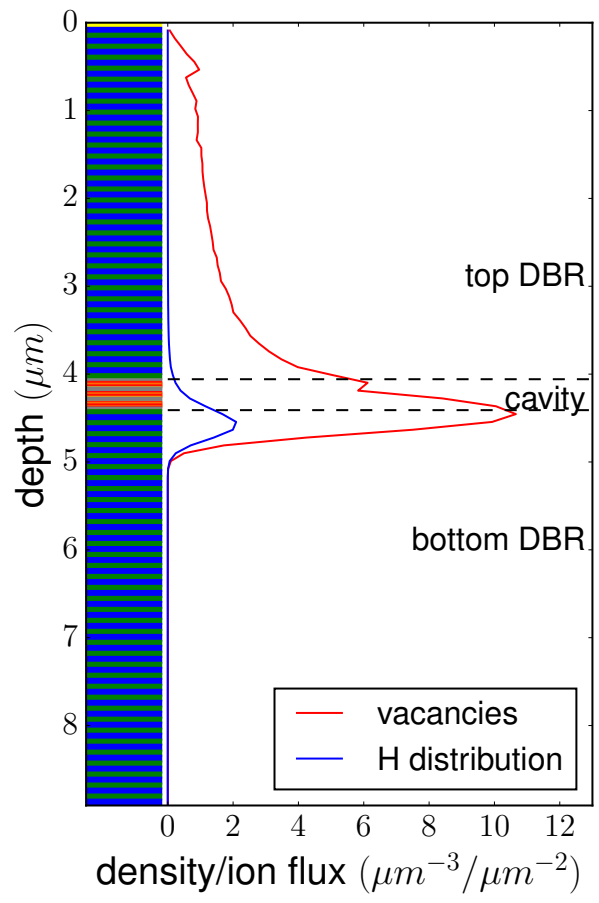

(b)

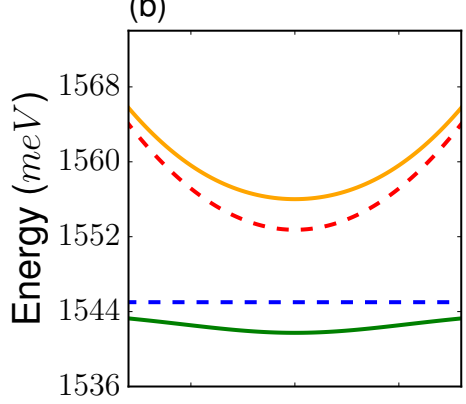

(d)

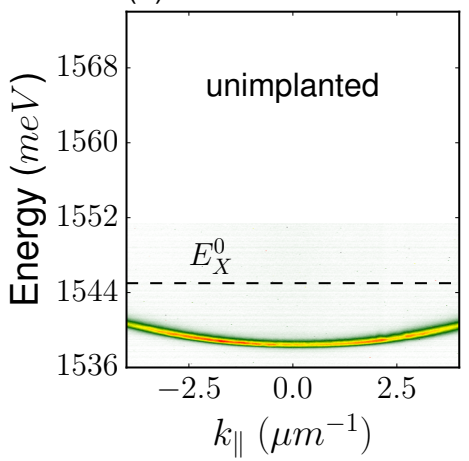

(c)

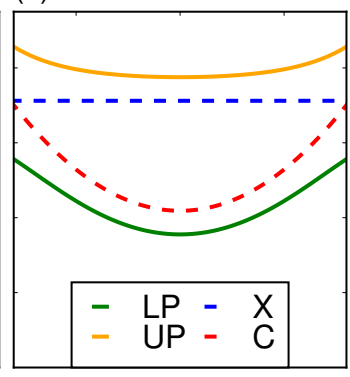

(e)

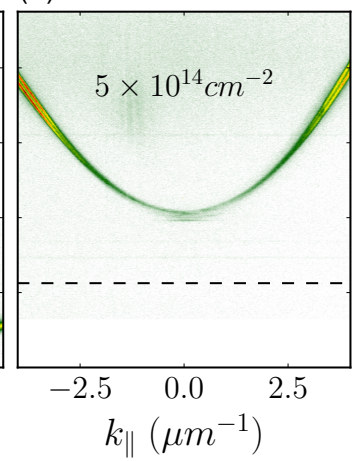

Figure 7. (a) shows the calculated ion and induced vacancy distribution at the optimal ion energy for a specific microcavity structure in order to localize the vacancies to the cavity region. As the exciton energy $E_{X}^{0}$ is increased in energy with increasing dose, an initially blue-detuned LP (b) will also shift to higher energy (c), while its mass decreases as a result of the shift towards negative detuning. Experimentally, an initially blue-detuned sample (d) after implantation with a dose of $5 \times 10^{14} \mathrm{~cm}^{-2}$ ions at $440 \mathrm{keV}$ (e), shows exactly these features.

equivalent to its amount of positive detuning. Provided the Rabi energy is also sufficiently large, implanting a low dose of protons into a polariton microcavity, with subsequent thermal annealing, will create a strong confining potential for a LP state with blue detuning.

Initial experimental studies of the technique on planar samples can reproduce the above described features. An originally blue-detuned sample [figure 7(d)], when implanted with a moderate dose, yields a polariton mode shifted by $\Delta E_{L P} \sim 15 \mathrm{meV}$, and shows a large reduction in the effective mass consistent with a shift of the polariton detuning into the red region [figure $7(\mathrm{e})]$.

3.1.4. Electrostatic Traps By applying electric fields to semiconductor quantum wells, the energy of the exciton can be tuned via the quantum confined Stark effect (QCSE). When locally applied to a sample, this effect can also be exploited to build flexible and elegant exciton traps. A major advantage of this technique certainly lies in the ability to electrically manipulate the trapping potential. For weak electric fields $F$, the shift of the exciton energy due to the QCSE can be expressed as:

$$
\Delta E=\beta F^{2}
$$


where $\beta$ is the polarizability of the quantum well excitons. Note that, for indirect excitons in coupled QWs (these are excitons where the electron is confined in one QW, and the hole in the other QW [79]), see figure 8(a,b) or for excitons in thick QWs, for high fields the energy shift is linear with the applied electric field $\Delta E=-e d_{\mathrm{eff}} F$, where $d_{\mathrm{eff}}$ denotes the separation between the QWs or the width of the thick QW. This trapping technique has been successfully exploited to localize (mostly indirect) excitons to study cold exciton gases in the presence of confinement. A cross-section of a design for such a trap (reproduced from [80]) is shown in figure 8(c).The electric field strength is laterally modulated due to shielding by an $\mathrm{SiO}_{2}$, which leads to the distinct formation of an electrostatic trap of QW excitons as can be seen by the deeply trapped indirect excitons in figure 8(d). This technology has been applied in similar forms for the creation of exciton traps with complex geometries [81], yielding the possibility to promote bosonic condensation phenomena [82].

A possible scheme for fabrication of electrostatic polariton traps is seen in figure 8(e), which shows semi-transparent contacts fabricated on top of a doped microcavity. Since the QWs are located in the intrinsic region, moderate applied voltages of a few hundred $\mathrm{mV}$ should suffice to shift the QW exciton emission up to several meV. It is interesting to note that, while the QCSE has been studied for exciton-polaritons in the linear regime [83, 84, 85], certain peculiarities related to phonon enhanced tunneling effects between nearby QWs and carrier screening were observed recently $[86,87]$. Under specific circumstances, in particular if diffusion of the reservoir is restricted, these effects indeed lead to a blueshift of the polariton resonance in the presence of the electric field, which may present a complication for the realization of electrostatic polariton traps.

An intrinsic limit to the tuning range of the exciton emission is the carrier tunneling out of the finite QW barriers. Furthermore, in order to laterally trap excitons, the QCSE has to be locally applied via a finite-size gate. As a result, lateral electric fields will evolve in the QW [88], which can also lead to a dissociation of the excitons. Despite the fact that there still is no conclusive demonstration of an electrostatic polariton Stark trap to date, the development of this technique would be highly advantageous, in particular because of its flexibility. A combination of electrostatic trapping or tuning with other confinement techniques described later in this article could yield the possibility to engineer reconfigurable polariton landscapes with unprecedented properties.

3.1.5. Confinement provided by the excitonic reservoir The carrier and exciton reservoir, which is induced by the excitation laser, provides a natural way to create potentials for the exciton polaritons. This approach provides a very elegant and useful technique to confine, manipulate and steer polaritons by exploiting their strong Coulomb interactions. An initial demonstration of this effect was discussed in a one-dimensional configuration [90]. Here, the polaritons were already partly confined in a deeply etched wire cavity along the wire. The high-energy excitonic reservoir created by the laser pump (tuned far off-resonace from the polariton energy) provided an additional potential barrier due to the strong repulsive interaction with polaritons. As a consequence, the polaritons became confined in all spatial directions close to the edge of the wire [figure 9(a)]. Conveniently, the size of the pump- 

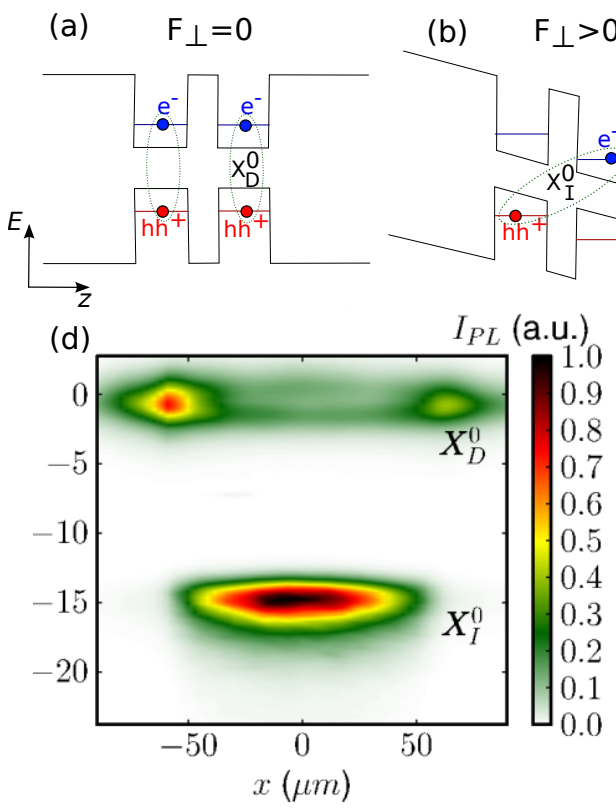

(c)

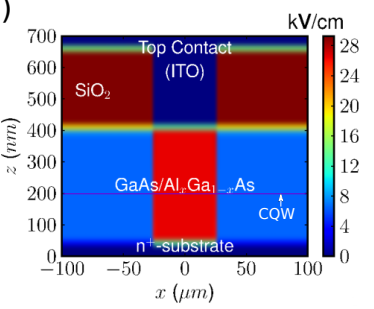

(e)

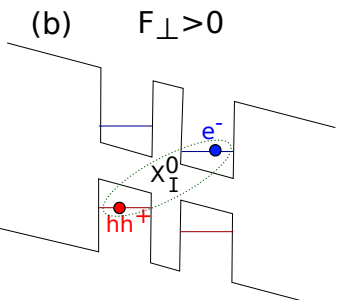

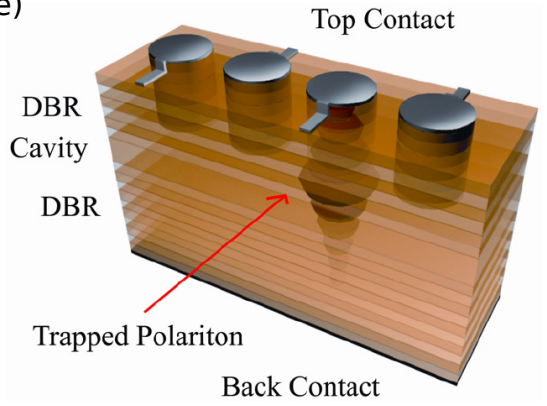

Back Contact

Figure 8. Electrostatic trapping of excitons and exciton-polaritons: (a) and (b) The exciton radiative transition without and with an applied voltage, the latter with long-lifetime indirect excitons $X_{I}$. (c) A cross-section of a trap design showing a modulation of the electric field strength felt by the excitons due to shielding by an $\mathrm{SiO}_{2}$ layer, and (d) the experimental realization of indirect exciton trapping. These figures are reproduced from [80]. Copyright (2011) by The American Physical Society. (e) Proposed trap for microcavity exciton-polaritons: The microcavity is locally capped by a semitransparent contact which simultaneously acts as a gate. Once a small bias is applied, the polariton energy is lowered underneath the trap, and an attractive potential is created. The figure is reproduced with permission from [89]

induced trap can be modified by changing the location of the pump spot on the wire cavity. In a similar way, polaritons can be confined all-optically by choosing smart geometries of the excitation laser beam. By using spatial light modulators to shape the optical excitation, ring shaped confinements [91, 68] [see figure 9(b)], confinements created by multiple pump spots $[66,92,91]$, and controlled flow patterns in more complex landscapes were generated [93]. Long-lifetime polaritons in large-area optically-induced ring traps are emerging as a platform for studies of fundamental properties of polariton condensation largely decoupled from the excitonic reservoir [94].

The concept of structuring the non-resonant pump profile to produce a targeted mode of the condensate has been further extended in [69] to demonstrate polariton condensates with a defined chirality. There, the pump beam has been structured via a metal amplitude mask with six pin-holes, which either were misaligned with respect to the laser beam, or structured in a chiral arrangement. As a result, trapped steady-state vortices were produced in the center of the six excitation spots, as confirmed by real space interferometry [see figure 9(c-e)].

The technique of controlling the environment for polariton condensate via pump shaping has proven extremely useful for the manipulation of the polaritons' properties due to its flexibility, however the depth of the provided confinement (or the potential height) is limited 
by the strength of the polariton-exciton repulsive interaction, which is of the order of $1 \mathrm{meV}$.
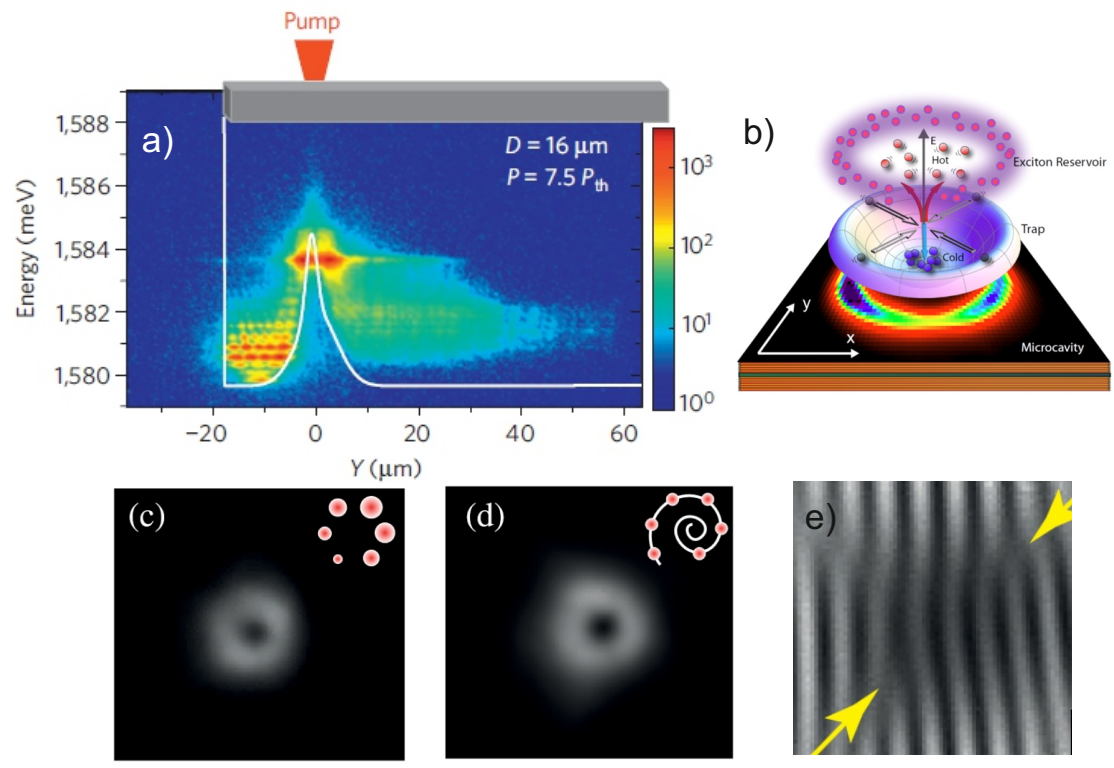

Figure 9. All-optical trapping of polaritons with the pump laser. (a) The polaritons are already confined in a one-dimensional microwire. The excitation laser spot provides the remaining boundary, and a zero-dimensional polariton condensate is formed close to the edge of the wire. The figure is reproduced from [90] (b) With a ring-shaped excitation laser spot, it is possible to create and confine a condensate in the center. The figure is reproduced from [68]. Copyright (2013) by The American Physical Society. (c-d) Method to create chiral structures by tailoring the exciton reservoir with a structured pump beam, by misalignment (c) and deliberatly designing the pump structure (d). Both techniques create a charge on vortex via non-resonant pumping. e) Interference image with a characteristic fork pattern, indicating the presence of a vortex. (c-e) are reproduced from [69]. Copyright (2014) by The American Physical Society.

3.1.6. Low-dimensional active material In order to complete this section, we will briefly review some examples of creating microcavity polaritons by directly using low-dimensional gain material instead of two-dimensional quantum wells subject to lateral trapping. In this discussion, we will limit ourselves to single semiconductor quantum dots on GaAs. A system composed of a single QD and a microcavity can be described by the single-particle JaynesCummings Hamiltonian [95], giving rise to an energy ladder structure which adds strong nonlinearities on the single photon level. For this reason, QD-polaritons are usually studied in the framework of isolated quantum systems, similar to atoms in high finesse microcavities [96]. Naturally, the possible application of QD-polaritons fundamentally differ from the QW-case.

Successful realizations of QD-polariton systems were first reported in [97, 98, 99] for various photonic resonator geometries. The first successful demonstration of true quantum effects in such systems (i.e. sub-poissonian emission statistics) in fundamental contrast to quantum well microcavity polaritons, was reported by [100] and [101]. Signatures of the 
photon blockade (or QD-polariton blockade), which is a fundamental effect that occurs due to the single photon non-linearity of the Jaynes-Cummings ladder system (see [102]), were observed in [103]. The electrical injection [104], electro-optical manipulation [105], and nonlinear emission [106] have been reported.

While the strong exciton localization in the quantum dot structures has many advantages due to the inherently very large non-linearities on the single- to few photon or exciton scale (leading to single photon emission and the polariton blockade effect), its main drawback lies in the poorly controlled nature of the fabrication process. It is still very challenging to gain control over the properties of single quantum emitters to such an extent, that they can be, e.g., arranged in arrays $[107,108]$ with designed spectral properties [109]. In this respect, a QW-polariton system, if it could be designed in such a way that nonlinearities are strongly enhanced, would be highly preferable.

\subsection{Trapping of polaritons via their photonic part}

Complementary to the discussion on the excitonic polariton trapping, in the following we will consider techniques to confine polaritons via their photonic part. Due to the relatively large extent of the photonic wavefunction, quantization and finite size effects can be observed for relatively large structures on the order of the polariton wavelength. This gives rise to 'quasi zero dimensional' exciton polaritons in structures with lateral dimensions on the order of $1-10 \mu \mathrm{m}$. Three of the most frequently exploited techniques, namely etching micropillars, defining shallow mesas and depositing metal masks on top of the two dimensional microcavity are sketched in figure 10, and will be discussed in more detail in the next section.

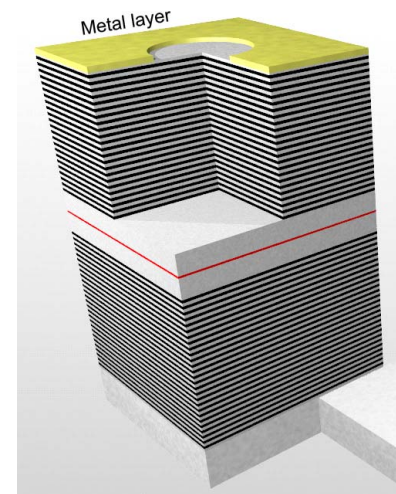

a) Thin film metal trap

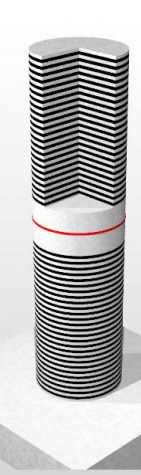

b) Micropillar

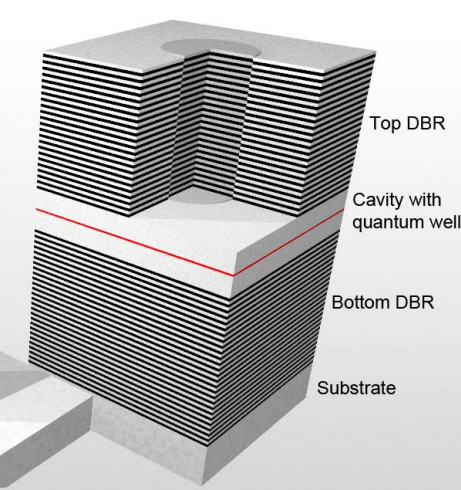

c) Textured cavity

Figure 10. Schematics of commonly applied techniques for spatial confinenement of excitonpolaritons via the photonic part: (a) depositing metal films on the top DBR layer, (b) etching micropillars and (c) defining shallow mesas in the cavity.

3.2.1. Natural photonic disorder traps In many microcavities, polariton localization can inherently occur in so-called photonic disorder potentials. These potentials can arise, e.g., due to natural local elongations of the cavity at the position of crystal defects and provide 
an optical confinement. It has also been shown, that the condensation of polaritons in a planar two-dimensional cavity is likely to occur in such natural potentials [110]. An accurate characterization of the formation of polaritonic states in natural traps was performed in [111]. Interestingly enough, these defect-induced traps are typically of a Gaussian shape, which is predicted to promote confined Q-factors strongly exceeding those of their mesa (vertical sidewall) counterparts for comparable mode volumes [112]. This makes such natural crystal defect traps highly appealing for the demonstration of large polariton nonlinearities and even photon blockade effects [113]. However, the scalability of this approach is strongly limited due to the randomness of these defects. In a later subsection, we will discuss in detail a technology enabling to implement similar structures in a microcavity in a fully controlled manner.

3.2.2. The metal mask technique A comparatively simple, yet very efficient method to create polariton confinement in a grown microcavity structure is the deposition of metal films on the sample surface. The metal layer changes the boundary conditions of the electromagnetic field with respect to the semiconductor-air interface, and creates an optical node. As a result, a modest shift in the energy of the optical resonance can be observed (on the order of $0.1-1 \mathrm{meV}$ ), which leads to an effective photonic confinement. The beauty of this approach certainly lies in its simplicity, and many pioneering experiments with polaritons in periodic potential landscapes were initially carried out in such samples. Examples include the first demonstration of a polariton condensate at higher-order Bloch bands in one-dimensional arrays [25] and the formation of polariton condensates in d- and f-states [18], as well as the condensation of polaritons close to Dirac points in triangular configurations [114]. A compilation of results obtained in the square lattice geometry is shown in figure 11 . As can be seen in figure 11(b), this technique yields relatively shallow lattice potentials $\left(\sim 10^{-2}-10^{-1}\right.$ $\mathrm{eV}$ ), and therefore the full energy gap, e.g., that between the first and the second band shown in figure 4, does not open.

An important extension of this approach is related to the excitation of the so-called Tamm plasmon (TP) states at semiconductor-metal interfaces. Localized TPs can evolve at the crystal surface at the interface between a periodic dielectric structure and a metal layer [115], and can be directly optically excited. The electric field distribution of such a TP decays into the periodic DBR structure, with a significant field enhancement close to the semiconductormetal interface. It is rather straightforward to couple TP modes to matter excitations, and the formation of Tamm plasmon exciton-polaritons has been reported $[116,84,117]$. In stark contrast to the deposition of the metal on top of a DBR, the lateral confinement provided by the Tamm plasmon can be significant [118].

For both methods, the Tamm plasmon approach or the metal mask deposition (which can also be considered as evanescent coupling of a Tamm state to a cavity photon), one has to compromise between the depth of the polariton confinement and the cavity Q-factor. While the Q-factor increases with the number of mirror pairs deposited onto the top DBR segment, the confinement depth decreases at the same time. This issue renders this approach incapable of providing spatial confinement for high-quality DBR based microcavities. For instance, 
for a sample with 16/20 AlGaAs/AlAs mirror pairs and a Q-factor on the order of 2000, a confinement depth of about $200 \mu \mathrm{eV}$ was experimentally extracted [119]. This number would be reduced to a value between $10-50 \mu \mathrm{eV}$ for a sample capped by more than 25 mirror pairs, as can be determined by a standard Transfer Matrix calculation.
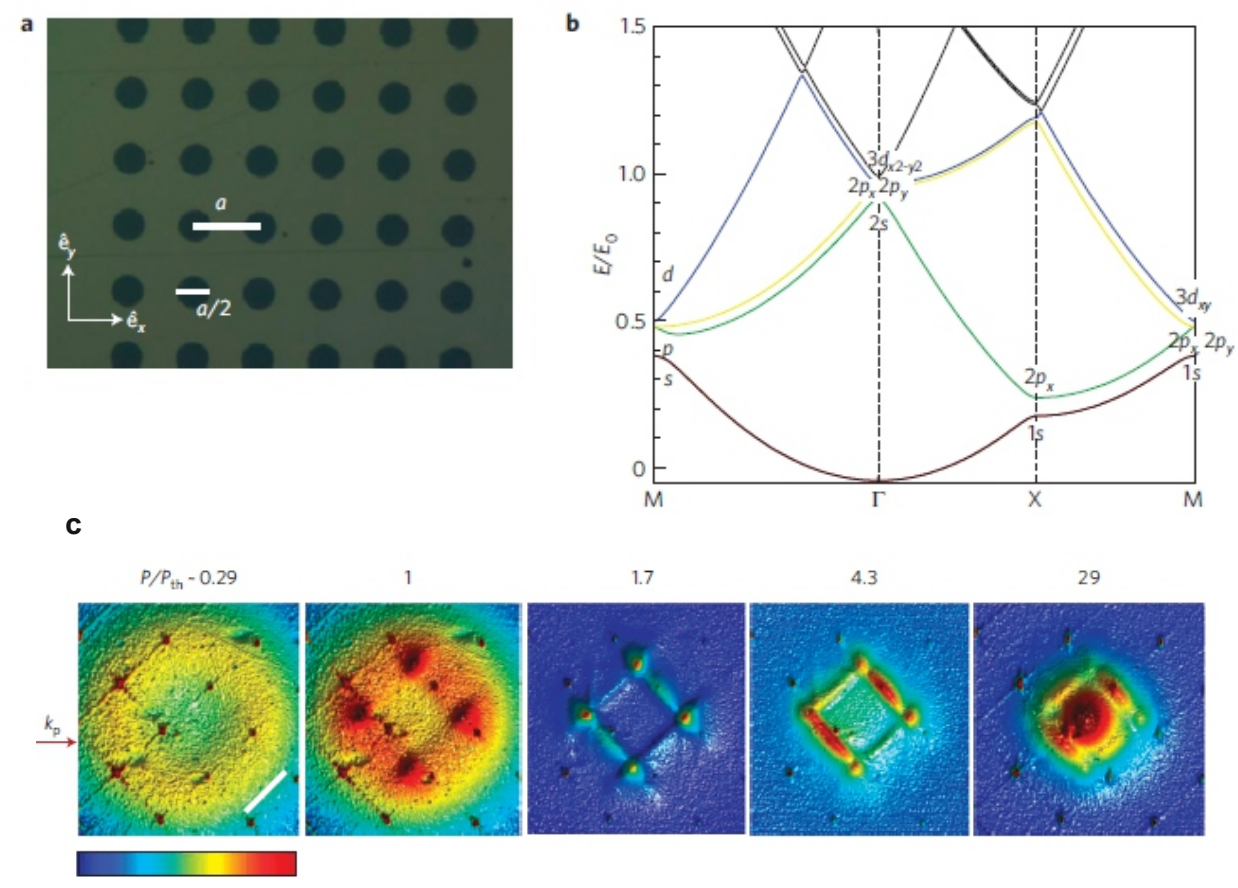

Figure 11. Confinement of polaritons by deposition of thin metal layers on a microcavity: (a) Photograph of a metal layer with micron sized circular holes forming a square lattice arrangement. (b) Calculated energy-momentum dispersion relation for the lattice geometry in (a) and a shallow potential [cf. figure 4(a) for the deep lattice potential].(c) Demonstration of the condensation of polaritons close to the high-symmetry points in the BZ, starting with a condensate at the characteristic M-point of the BZ. Reprinted with permission from [18].

3.2.3. Etched micropillar cavities Deep etching of micropillars in the sample is perhaps the most straightforward approach to lateral confinement of a photon in a DBR microcavity. Additionally, diffusion and propagation of excitons is also limited to the pillar size, which has some important implications on the performance of polariton lasers in the pillar geometry [120]. In this geometry, the optical field is laterally confined by the semiconductor-air interface. This approach has been widely exploited to fabricate vertically emitting lasers and efficient single photon sources $[121,122,123]$. In order to realize such structures, typically optical lithography or electron beam lithography is employed to define an arbitrary shape of the pillar in a photo- or electron beam sensitive resist. This resist can be either directly used as an etch mask, or the latter can be evaporated after the lithography step. Plasma etching has been proven to facilitate the realization of nearly perfect posts with vertical sidewalls in the GaAs system. A typical example of such a micropillar with a diameter of $2 \mu \mathrm{m}$ is shown in figure 12(a). Note that careful optimization of the etching procedure (e.g. reactive 
ion etching with $\mathrm{Cl}_{2}$ /Ar plasma) can lead to almost perfectly vertical and smooth sidewalls, which is crucial when scattering and diffraction losses should be kept minimal [37]. With this technique, it is possible to realize DBR-based zero dimensional microcavities with Qfactors exceeding 250000 [37]. The finite physical size of this micropillar results in a strong optical confinement for the microcavity photon as a result of the huge difference between the refractive index in the structure $(n \approx 3.5)$ and its surroundings $(n \approx 1)$. Hence, the optical mode spectrum splits into a set of characteristic waveguide modes. A characteristic photoluminescence spectrum from such a micropillar cavity is shown in figure 12(b). The parabolic cavity resonance splits into a set of discrete, confined photonic modes, arising from the circular waveguide geometry (see, e.g., [124, 125, 126]).
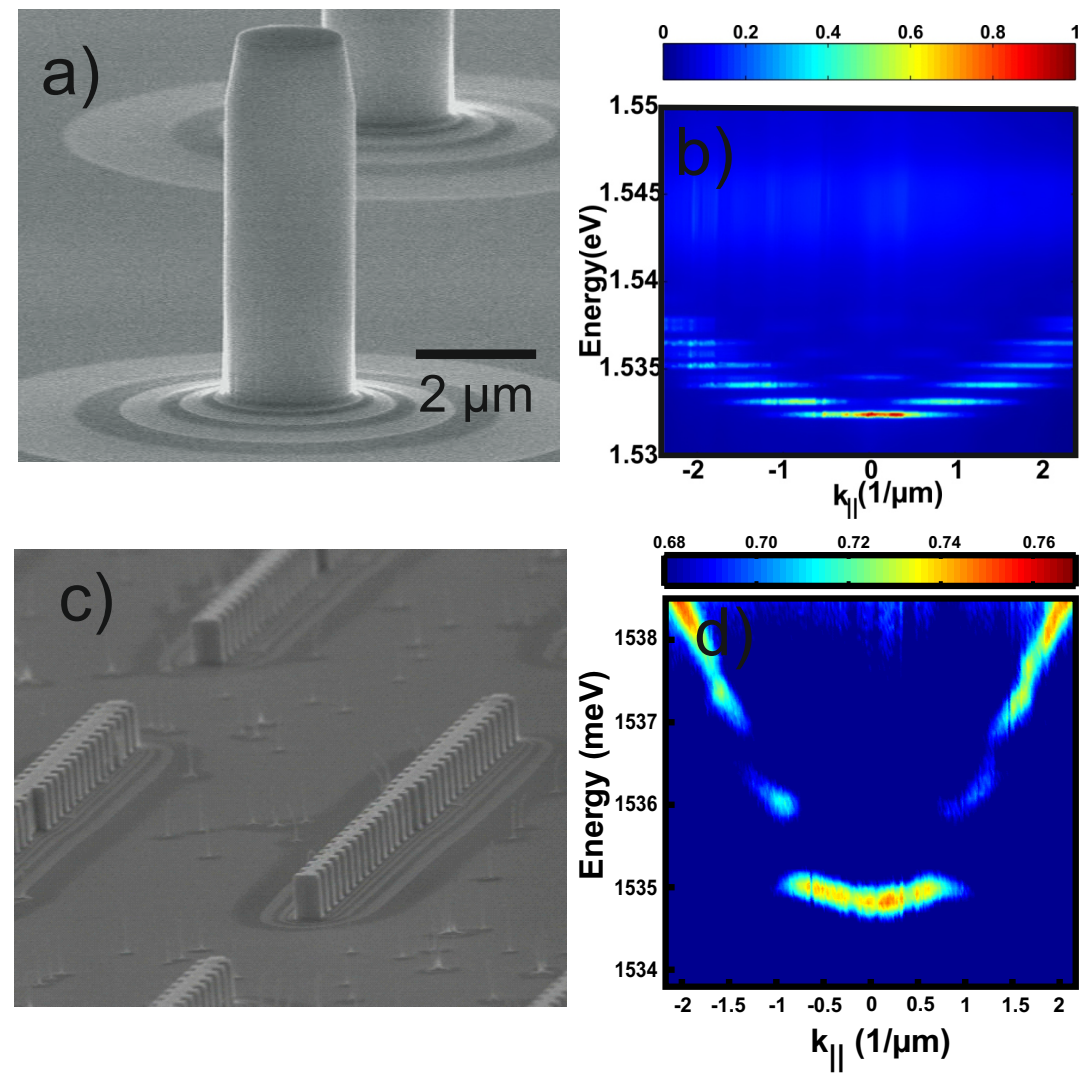

Figure 12. (a) SEM image of a micropillar cavity, etched into a DBR based structure. (b) Photoluminescence spectrum of the optical modes in a micropillar device. (c) SEM image of a 1D arrangement of rectangular micropillars, resembling a modulated microwire. (d) The energy-momentum dispersion of the modulated wire features a band gap spectrum with distinct gaps at the edges of the BZ.

Despite the brute force approach to lateral optical confinement, and associated degradation of the QW properties due to surface related effects, strong coupling effects in such micropillars were successfully demonstrated by Gutbrod et al. [127], where the characteristic anticrossing between optical and electronic resonance was fully mapped out 
via temperature tuning. In this work, the effects of light-matter coupling were amplified by an applied magnetic field, which enhanced the oscillator strength of the integrated QWs and lead to an increase of the light-matter coupling strength. Subsequent benchmark experiments include the first demonstration of polariton condensation in a micropillar cavity in the II/VI system [128] and in the GaAs system [129].

A particular advantage of defining polariton potentials via lithography and etching lies in the simplicity of the approach. It is comparably easy to create almost arbitrary potential profiles and to study the behavior of the quantum gas in such tailored environments. In this spirit, polariton condensates in one dimensional microwires have been generated and their propagation and coherence properties were investigated [90, 130, 131]. Furthermore, the condensation of polaritons in microwires with a modulated sidewall [in other words, a onedimansional array of coupled rectangular micropillars, see figure 12(c)] has been reported [54]. The overlap between adjacent pillars leads to photonic coupling, resulting in photonic bands with well pronounced gaps at the edge of the BZ [figure 12(c)], which has been investigated already in the early works by Dasbach et al. [132]. As we will detail later, the peculiar band structure of such an array of coupled micropillars featuring full bandgaps and associated negative effective mass regions gives rise to interesting condensation phenomena, such as the formation of gap solitons [54].

Owing to the high flexibility and maturity of the electron beam lithography and etching technologies, two-dimensional arrays of overlapping micropillars can also be realized rather straightforwardly. Initial measurements of the photonic band structure in two-dimensional micropillar lattices were reported by Bayer et al. [133]. An example of the implementation of a polariton condensate in a hexagonal potential environment is shown in figure 13(a). The structure features an optical potential landscape resembling the graphene-type lattice (socalled honeycomb structure), which is also reflected in the optical properties of the emitted light. The most striking property of this lattice configuration is the appearence of the Dirac points at specific locations in the BZ. A Dirac point is characterized by its linear dispersion of mass-less particles. The condensation of polaritons in the vicinity of a Dirac point is demonstrated in figure 13(b).
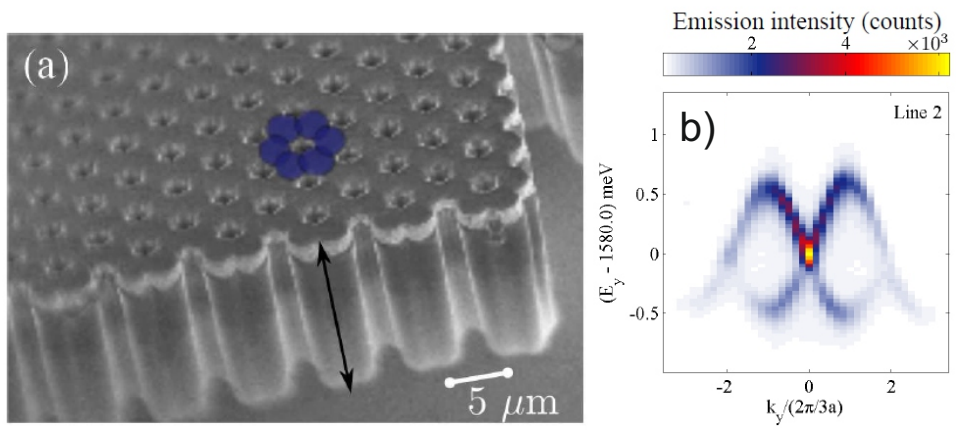

Figure 13. (a) SEM image of an array of micropillars aligned in a honeycomb lattice configuration. (b) The potential landscape gives rise to Dirac cones with a linear Energymomentum dispersion relation. The condensation of polaritons near such a Dirac point is discussed in [134]. Copyright (2014) by The American Physical Society. 
Furthermore, the maturity of this approach has already led to the first generation of polaritonic logic devices designed for integrated photonic experiments. These devices take advantage of the possibility to direct the flow of polaritons along etched channel structures over macroscopic distances, and to manipulate them via optical or electric fields, resulting in the successful implementation of switches, interferometers, and tunneling diodes $[16,15,135]$.

The ease of the micropillar approach comes along with a significant disadvantage. In order to provide a strong mode confinement, the structures are commonly etched through the active region (the QWs). As a consequence, nonradiative recombination of the excitons at the surface can occur, and furthermore etching through the QWs is accompanied by degradation of the emission. This effect can be pronounced, and is visible for instance in the spectrum of the etched micropillar in figure 12(b), where the emission from a strong uncoupled background resulting from etching through the active region can be seen. Therefore, less destructive methods to incorporate deep and flexible polariton confinements into polariton landscapes are highly desirable.

3.2.4. Photonic crystals and hybrid approaches Similarly to the fabrication of micropillar cavities to confine polaritons, photonic crystal nanocavities can also provide a tight optical confinement with the smallest mode volumes. This technique is particularly popular for the fabrication of microlasers with the smallest footprints and coupled systems with quantum dot emitters, due to the small mode volumes and the resulting large light-matter coupling strength $[98,101]$. To date, there are only few demonstrations of polariton lasers in conventional photonic crystal nanocavities [136] [figure 14(a)]. A (hybrid) photonic crystal approach which fully circumvents the etching of the active medium has been introduced by Zhang et al. [137, 138]. Similarly to the methods used to build flexible vertically emitting microlasers [139], only the upper DBR mirror is replaced by a highly reflecting sub-wavelength high contrast grating (HCG), which is a broadband crystal mirror. A sketch of the resulting structure, which has been proven to promote polariton lasing features [137] is shown in figure 14(b). This work has been successfully extended by coupling of such polaritonic boxes to photonic molecules and one-dimensional superlattices [140], which demonstrates the versatility and potential of the approach.

In the HCG-cavity, strong coupling conditions can only be maintained underneath the finite sized grating. This finite size effect results in an effective in-plane confinement of polaritons, restricted to the dimension of the HCG. Another approach to define zero dimensional polaritons by reducing the lateral area was introduced in [141]. The authors designed a single mode fiber with an integrated Bragg grating, which they used to replace the top DBR. While the curvature of the fiber tip defines the mode volume in such a cavity, the resonance frequency of the resulting hybrid device can be almost arbitrarily varied by varying the distance between the fiber tip and the sample surface. This elegant techniques promises ultra-high Q-factors, low mode volumes and, consequently, is a prime candidate for investigating non-linearities on the single polariton scale. A conceptually similar concept was introduced in [142], where the authors demonstrated strong coupling conditions in a so-called 

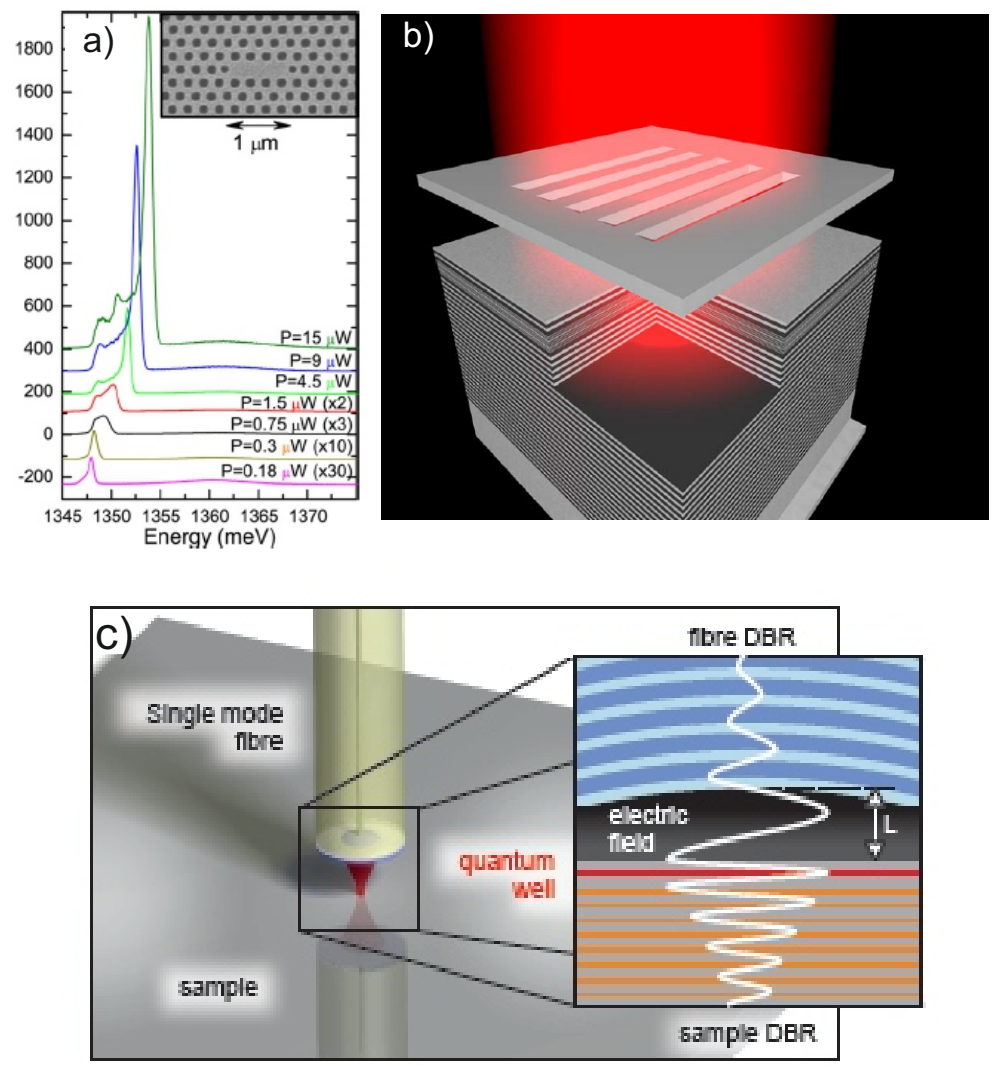

Figure 14. (a) Polariton laser based on a GaInP L3 photonic crystal nanocavity (figure reprinted with permission from Azzini et al. [136]). (b) Sketch of a hybrid photonic crystalDBR polariton laser. (c) Hybrid fiber cavity polariton laser. The figure is reproduced from [141]. Copyright (2015) by The American Physical Society.

open cavity approach. Here, one DBR is attached to piezo actuators which allow to tune the physical cavity length, while a tight optical confinement is introduced by locally shaping the curvature of the mirror.

3.2.5. Etch-and-overgrowth technique As indicated in section 3.2.1, a shallow modulation of the cavity length, e.g. via a crystal defect initiated by a droplet, is sufficient to create a well defined, deep lateral photonic confinement [111]. A straightforward lithographic implementation of this concept leads to creation of polariton mesa traps. These traps have been first reported by El Daif et al.[143] and where subsequently theoretically analyzed with respect to the feasibility to observe polariton BEC states in lattice configurations [50]. Although the realization of such mesa traps is certainly more challenging compared to other approaches, including etching of regular micropillars or deposition of metal masks, the approach is very appealing due to the following significant advantages: (i) the confinement depth can be tuneable in a wide range, mainly by adjusting the height of the defect and the light-matter detuning; (ii) inter-site coupling between neighbouring traps is readily controllable; (iii) surface recombination effects from etching through the active medium are fully avoided. These traps are commonly realized by texturing of the cavity layer of a Fabry- 
Perot-microcavity structure into elongated (trap) and regular (planar) regions. As the cavity resonance condition is fulfilled for photons with a longer wavelength, the trap region acts as an attractive potential for photons. If the lateral dimension of the trap region is comparable to the wavelength of the cavity-photons or polaritons, discrete 1D or 0D modes evolve in the system.

Such a structure, which is sketched in figure 15(a), can be realized e.g., via molecular beam epitaxy (MBE) growth in a three-step etch-and-overgrowth procedure. First, the bottom DBR mirror and the cavity layer including all optical active regions (QWs) are grown. In the case of an AlAs-cavity including GaAs-QWs, a thin GaAs-capping layer should be grown on top of the AlAs-cavity-layer to protect the sample from oxidation [51], and ideally be placed at a node of the optical field to circumvent absorption. This structure then gets transferred out of the MBE-reactor for patterning of the MC via lithography and etching prior to epitaxial overgrowth. Routinely, electron beam lithography is used to define the traps. However, existing techniques for creating three dimensional nanostructures [144, 145] have a great potential for implementation of advanced geometries. In fact, the shallow elongation of the MC spacer is preserved even after deposition of the several microns thick top DBR mirror and can be fully resolved on the sample surface, e.g. via atomic force microscopy, as seen in figure 15(b).
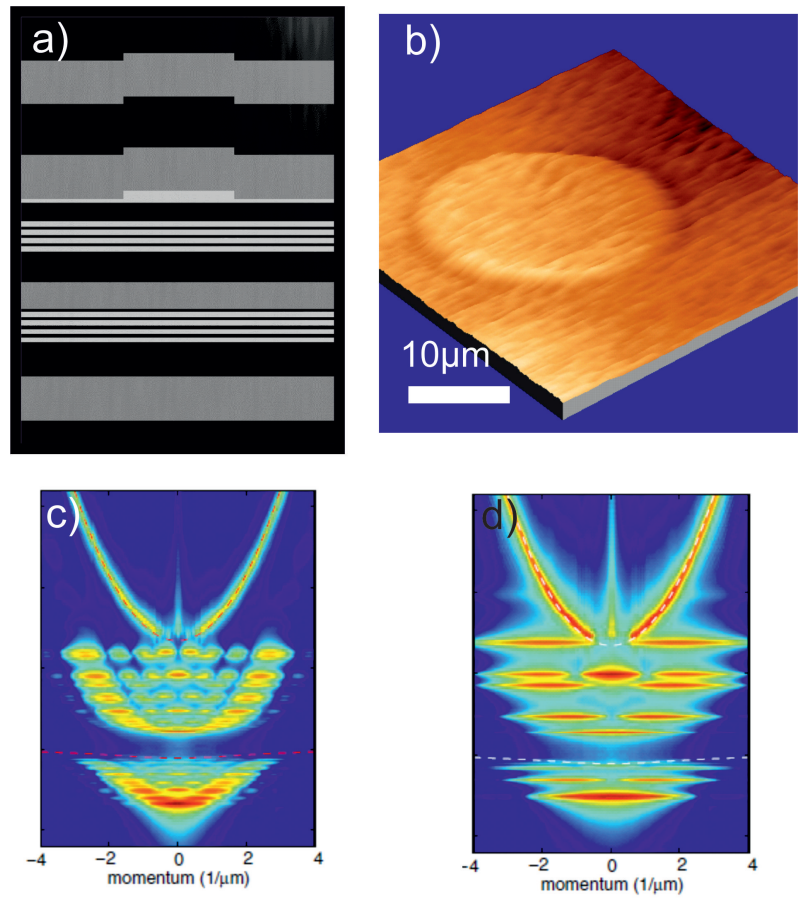

Figure 15. (a) Schematic drawing of the mesa trap structure to provide a lateral confinement for polariton condensates. (b) AFM image of the surface of an overgrown trap. Simulation of the energy-momentum dispersion of a trap with a diameter of $8.6 \mu \mathrm{m}$ (c) and $3.6 \mu \mathrm{m}$ (d) respectively. Reprinted from [49], Copyright (2006) by The American Physical Society.

A simulation of the polariton energy-momentum dispersion in such a mesa is shown in figures 15(c )and (d). Due to the finite size effect, a strong mode quantization is evident, which 
scales with the size of the lateral confinement. Emission from the planar microcavity persists in the background, however this emission can be significantly blue-shifted by increasing the etch depth.

The confinement of polaritons in such a structure can be rather accurately modelled by calculating the single particle energy levels in a finite, cylindrically symmetric (polariton) potential well. The (lateral) well depth is given by the height of the mesa (and the corresponding shift in the Bragg resonance energy). Then, the Schrödinger equation can be solved for a particle with the polariton effective mass $m_{L P}$, yielding the discrete energy levels which can be seen in figures 15 (c) and (d) for a diameter of 10 and $3.6 \mu \mathrm{m}$ [49]. The low dimensional confinement, which can be designed in a wide range, can be utilized to engineer the scattering properties in such structures. A detailed discussion of the relaxation processed in such low-dimensional polariton structures is given in [146]. Noteworthy, it is found that the phonon relaxation between localized levels in the trap is enhanced (compared to 2D samples) as a consequence of momentum space selection rules, whereas energy transfers between 2D and $0 \mathrm{D}$ polaritons are strongly suppressed. Consequently, it is suggested that, under nonresonant excitation conditions, the $0 \mathrm{D}$ polariton states have to be directly populated from the exciton reservoir, rather than from the $2 \mathrm{D}$ polaritons.

The first demonstration of polariton condensation under non-resonant pumping in a mesa was discussed quite recently in [51], where the authors investigated a single trap with a diameter of $6 \mu \mathrm{m}$ and a depth of $\sim 30 \mathrm{~nm}$.
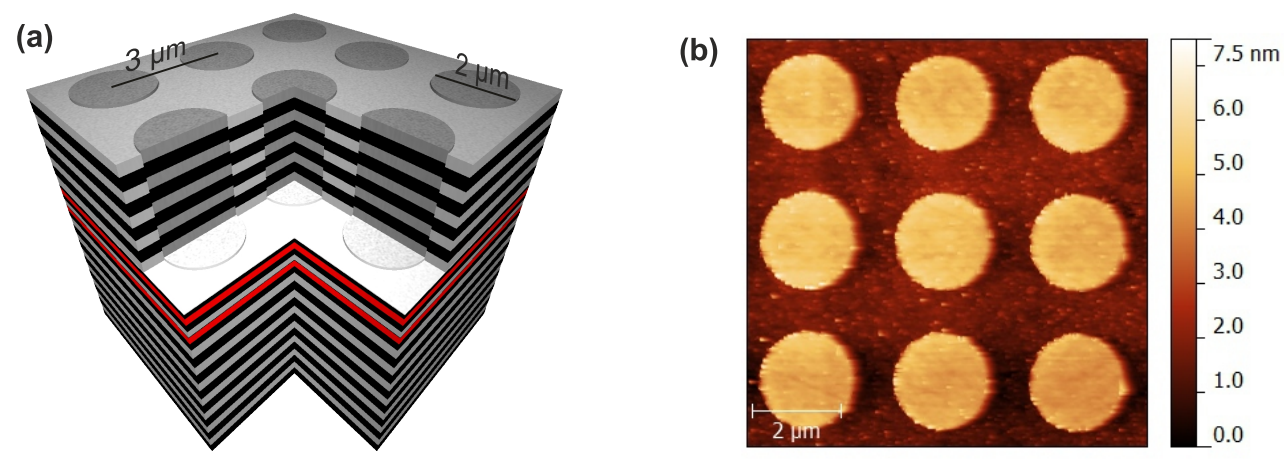

Figure 16. Polaritons in a square lattice potential landscape. (a) Schematic drawing of the investigated square lattice structure consisting of buried polariton traps. (b) Atomic force microscopy image of the etched cavity surface, depicting the morphology of the mesa structure.

The full potential of this technique becomes obvious, when the traps are arranged in more complex geometries, such as square lattice arrangements (figure 16). The polariton wave functions can penetrate into the barrier leading to evanescent photonic coupling between neighboring sites which is accompanied by the formation of a band structure. Using the linear exciton-photon coupling Hamiltonian[38], it can be shown that the structure of the energy bands imposed on the cavity photon mode by the periodic potential translates into the band structure of the polariton spectrum [50] (see section 2.5). 
A comparison between experiment and theory is shown in figure 17(a), recorded for a square lattice with a constant of $3 \mu \mathrm{m}$ below condensation threshold from the $\Gamma$-point to the $\mathrm{X}$-point of the BZ. The wave-function overlap is sufficient to form distinct bands for the three lowest energy levels, which can be perfectly reproduced by a model with realistic parameters: $V_{L}=3.2 \mathrm{meV}, m_{L P}=5.6 \times 10^{-5} m_{e}$.
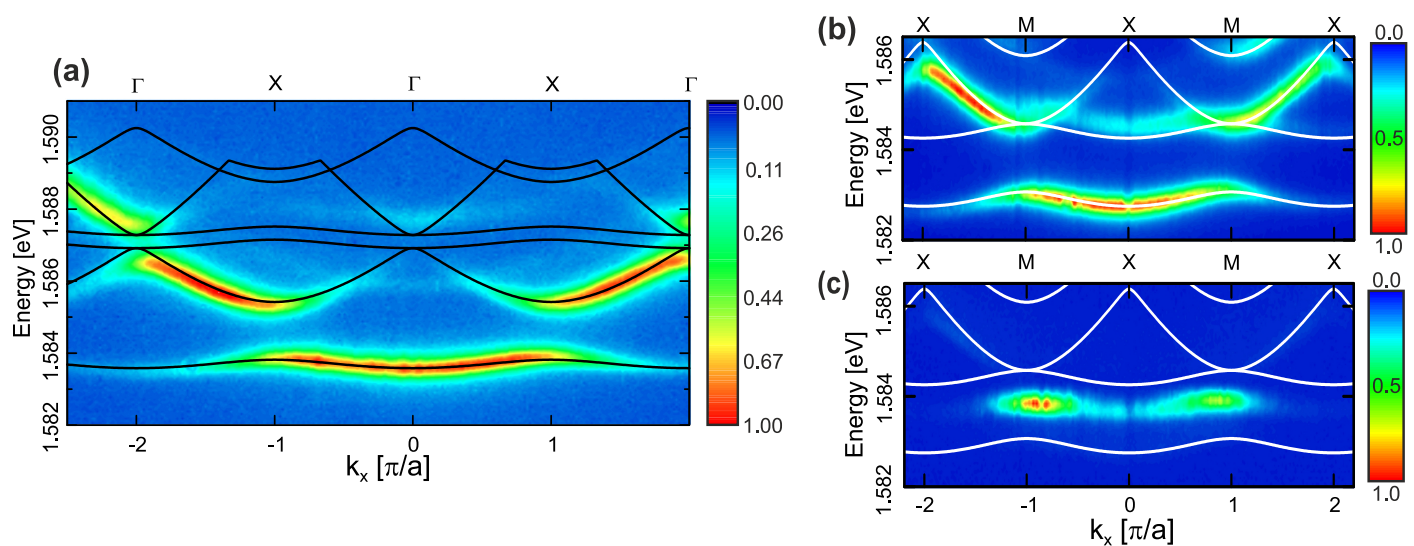

Figure 17. Polariton condensation in a square lattice (a) Photoluminescence spectrum of a square lattice with a lattice constant $a=3 \mu \mathrm{m}$. The axis shows the momentum in units of the BZ, while the upper axis marks the symmetry points. The band structure calculated theoretically is plotted in black lines. (b) Zoom onto the band gap region of a square lattice with a lattice constant $a=3 \mu \mathrm{m}$ recorded along the X-M symmetry direction revealing a complete gap between the $\mathrm{M}$ and X symmetry points of the first BZ. (c) For large pump power (1.3 times power at the condensation threshold $P_{t h}=5.7 \mathrm{~mW}$ ), polariton condensation in the full gap near the M-point is observed. The figure is reprinted with permission from [51]

The nonlinear characteristics of the polaritons in such a lattice is particularly manifested close to the M-point of the BZ, which is shown in the close up image of the gap between the $\mathrm{s}$ and p-band in our system, taken along the X-M symmetry direction below [figure 17(b], $P \sim 0.2 P_{t h}$ ] and above [figure 17(c), $P \sim 1.3 P_{t h}$ ] the condensation threshold. With increasing pump power, the condensate forms in the vicinity of M-points of the BZ blue shifts into the full gap of the linear spectrum. This behavior suggests the formation of a spatially localized twodimensional gap soliton state [147], also observed in the potentials created by surface acoustic waves [55]. These gap states are analogous to their 1D counterparts observed in modulated photonic wires [54] and arrays of mesa traps [56], however the essential requirement for their existence in 2D is the complete energy gap which, at lower energies, is only available at the M-point of the square lattice BZ. Although particular parameters of the microcavity and the polariton potential do affect the process of gap soliton formation, their spatial and spectral properties are rather universal. 


\section{Conclusions and outlook}

Owing to the rapid development of the techniques for manipulating light and matter waves on the scale of their characteristic wavelengths, it has become possible to engineer potential landscapes for light-matter particles in semiconductors. While state-of-the-art microcavities facilitate exciton-polariton propagation over hundreds of microns with the velocities close to the speed of light, the polaritons' hybrid nature allows for their efficient manipulation via electro-optical and all-optical methods.

The possibility to create polariton condensates in a variety of trapping geometries, to engineer and manipulate scattering mechanisms, to control propagation of polaritons in channels, and to form versatile periodic potentials, opens the way towards more advanced fundamental studies and applications of polariton physics.

One interesting opportunity, which we have touched in this review, can arise by utilizing polaritons in channel structures for integrated polaritonic schemes. Another interesting option involves exploiting polaritons in periodic potential landscapes to create polaritonic topological insulators [148, 149], which facilitate unidirectional propagation of polaritons in edge states protected from back-scattering by topology. While the investigation of topological effects in coupled light-matter systems is extremely appealing from the fundamental point of view, there is also a vast variety of possible applications arising from such effects.

Further improvements of trapping schemes at the sub-micron scale could enable the regime of polariton blockade, where non-linearities occur on the single polariton scale. Successful engineering of potentials allowing for single polariton localization is still a major challenge in the field, however it carries the promise to open new directions in the research field of quantum polaritonics.

Furthermore, the inherently lossy nature of the polariton system combined with the gain derived from the optical pump, offers a possibility to create and control potential landscapes with both real and imaginary part, and therefore to explore non-Hermitial quantum physics. This possibility has already been exploited to observe exceptional points and topological Berry phase in a polaritonic quantum billiard [150], to demonstrate the novel effect of weak polariton lasing in a one-dimensional periodic potential [151], and to load exciton-polaritons into a flat energy band of a one dimensional 'Lieb' lattice potential [152].

Finally, practical applications of the fascinating effects described in this review clearly require a clean, reliable material platform which can support exciton-polaritons well above cryogenic temperature range. Utilizing materials with large exciton binding energies, such as organic semiconductors [153], GaN [154], and $\mathrm{ZnO}$ [155], or atomically thin layers of transition metal dichalcogenides [156] can hence lead to novel architectures in integrated photonics and optoelectronics based on coherent bosonic states created and maintained at room temperature. 


\subsection{Acknowledgments}

The authors thank A. Kavokin, I.A. Shelykh, I.A. Savenko, H. Flayac, T. Fink, T.C.H. Liew, A. Nalitov, N. Gregersen, N.Y. Kim, S. Brodbeck, R. Dall, B. Bradel, A. Schade, A. Löffler, A. Imamoglu, V.D. Kulakovskii, L. Worschech, A. Forchel for collaborations, uncountable discussions and support throughout all the years.

Expert technical support in sample preparation by M. Emmerling, A. Wolf, M. Wagenbrenner, S. Handel, S. Kuhn and T. Steinl is gratefully acknowledged. This work was funded by the State of Bavaria and the Australian Research Council. M.D. Fraser acknowledges funding by ImPACT Program of Council for Science, Technology and Innovation (Cabinet Office, Government of Japan).

\section{References}

[1] Kavokin, A.V. and Baumberg, J.J. and Malpuech, G. and Laussy, F.P. Microcavities. Oxford Science Puplications, (2007).

[2] Yamamoto, Y., Tassone, F., and Cao, H. Semiconductor Cavity Quantum Electrodynamics. Springer, (2002).

[3] Kasprzak, J. et al. Bose-Einstein condensation of exciton polaritons. Nature 443, 409-414 (2006).

[4] Anderson, M. H., Ensher, J. R., Matthews, M. R., Wieman, C. E., and Cornell, E. A. Observation of Bose-Einstein condensation in a dilute atomic vapor. Science 269, 198201 (1995).

[5] Balili, R., Hartwell, V., Snoke, D., Pfeiffer, L., and West, K. Bose-Einstein condensation of microcavity polaritons in a trap. Science 316, 1007-1010 (2007).

[6] Deng, H., Weihs, G., Santori, C., Bloch, J., and Yamamoto, Y. Condensation of semiconductor microcavity exciton polaritons. Science 298, 199-202 (2002).

[7] Tempel, J.-S. et al. Temperature dependence of pulsed polariton lasing in a GaAs microcavity. New Journal of Physics 14, 083014 (2012).

[8] Kasprzak, J. et al. Second-order time correlations within a polariton Bose-Einstein condensate in a CdTe microcavity. Physical Review Letters 100, 067402 (2008).

[9] Deveaud-Pledran, B. On the condensation of exciton-polaritons. JOSA B 29 (1995).

[10] Einstein, A. Quantentheorie des einatomigen idealen gases zweite abhandlung. Sitzungsberichte der preussischen Akademie der Wissenschaften , 3-10 (1924).

[11] Hohenberg, P. Existence of long-range order in one and two dimensions. Physical Review 158, 383 (1967).

[12] Schneider, C. et al. An electrically pumped polariton laser. Nature 497, 348-352 (2013).

[13] Liew, T. C. H., Kavokin, A. V., and Shelykh, I. A. Optical circuits based on polariton neurons in semiconductor microcavities. Physical Review Letters 101, 016402 (2008). 
[14] Liew, T. C. H. et al. Exciton-polariton integrated circuits. Physical Review B 82, 033302 (2010).

[15] Sturm, C. et al. All-optical phase modulation in a cavity-polariton Mach-Zehnder interferometer. Nature Communications 5 (2014).

[16] Gao, T. et al. Polariton condensate transistor switch. Physical Review B 85, 235102 (2012).

[17] Liew, T. C. H., Shelykh, I. A., and Malpuech, G. Polaritonic devices. Physica E: Low-dimensional Systems and Nanostructures 43, 1543-1568 (2011).

[18] Kim, N. Y. et al. Dynamical d-wave condensation of exciton-polaritons in a twodimensional square-lattice potential. Nature Physics 7, 681-686 (2011).

[19] Jacqmin, T. et al. Direct observation of Dirac cones and a flatband in a honeycomb lattice for polaritons. Physical Review Letters 112, 116402 (2014).

[20] Masumoto, N. et al. Exciton-polariton condensates with flat bands in a twodimensional kagome lattice. New Journal of Physics 14, 065002 (2012).

[21] Cirac, J. I. and Zoller, P. Goals and opportunities in quantum simulation. Nature Physics 8, 264-266 (2012).

[22] Carusotto, I. and Ciuti, C. Quantum fluids of light. Reviews of Modern Physics 85, 299 (2013).

[23] Takemura, N., Trebaol, S., Wouters, M., Portella-Oberli, M., and Deveaud, B. Heterodyne spectroscopy of polariton spinor interactions. Physical Review B 90, 195301 (2014).

[24] Takemura, N., Trebaol, S., Wouters, M., Portella-Oberli, M. T., and Deveaud, B. Polaritonic Feshbach resonance. Nature Physics (2014).

[25] Lai, C. W. et al. Coherent zero-state and $\pi$-state in an exciton-polariton condensate array. Nature 450, 529-532 (2007).

[26] Kim, N. Y. et al. Exciton-polariton condensates near the Dirac point in a triangular lattice. New Journal of Physics 15, 035032 (2013).

[27] Byrnes, T., Recher, P., and Yamamoto, Y. Mott transitions of exciton polaritons and indirect excitons in a periodic potential. Physical Review B 81, 205312 (2010).

[28] Verger, A., Ciuti, C., and Carusotto, I. Polariton quantum blockade in a photonic dot. Physical Review B 73, 193306 (2006).

[29] Liew, T. C. H. and Savona, V. Single photons from coupled quantum modes. Physical Review Letters 104, 183601 (2010).

[30] Bamba, M., Imamoğlu, A., Carusotto, I., and Ciuti, C. Origin of strong photon antibunching in weakly nonlinear photonic molecules. Physical Review A 83, 021802 (2011).

[31] Na, N. and Yamamoto, Y. Massive parallel generation of indistinguishable single photons via the polaritonic superfluid to mott-insulator quantum phase transition. New Journal of Physics 12, 123001 (2010). 
[32] Klingshirn, C. Semiconductor Optics. Springer, (2004).

[33] Weisbuch, C. and Vinter, B. Quantum Semiconductor Nanostructures. Academic Press, Inc., (1991).

[34] Masselink, W. T. et al. Absorption-Coefficients and Exciton Oscillator-Strengths in AlGaAs-GaAs Superlattices. Physical Review B 32, 8027-8034 (1985).

[35] Andreani, L. C. and Pasquarello, A. Accurate theory of excitons in GaAs-GaAlAs quantum wells. Physical Review B 42, 8928 (1990).

[36] Reitzenstein, S. et al. AlAs/GaAs micropillar cavities with quality factors exceeding 150.000. Applied Physics Letters 90, 251109 (2007).

[37] Schneider, C., Gold, P., Reitzenstein, S., Hoefling, S., and Kamp, M. Quantum dot micropillar cavities with quality factors exceeding 250,000. Applied Physics B 122, 1-6 (2016).

[38] Deng, H., Haug, H., and Yamamoto, Y. Exciton-polariton Bose-Einstein condensation. Reviews of Modern Physics 82, 1489-1537 (2010).

[39] Savona, V., Andreani, L., Schwendimann, P., and Quattropani, A. Quantum well excitons in semiconductor microcavities: Unified treatment of weak and strong coupling regimes. Solid State Communications 93, 733-739 (1995).

[40] Khitrova, G., Gibbs, H., Jahnke, F., Kira, M., and Koch, S. Nonlinear optics of normalmode-coupling semiconductor microcavities. Reviews of Modern Physics 71, 1591 (1999).

[41] Byrnes, T., Kim, N. Y., and Yamamoto, Y. Exciton-polariton condensates. Nature Physics 10, 803-813 (2014).

[42] Houdré, R. et al. Saturation of the strong-coupling regime in a semiconductor microcavity: Free-carrier bleaching of cavity polaritons. Physical Review B 52, 7810 (1995).

[43] Kavokin, A., Malpuech, G., and Gil, B. Semiconductor microcavities: towards polariton lasers. MRS Internet Journal of Nitride Semiconductor Research 8, e3 (2003).

[44] Petrov, D., Holzmann, M., and Shlyapnikov, G. Bose-Einstein condensation in quasi2D trapped gases. Physical Review Letters 84, 2551 (2000).

[45] Petrov, D., Shlyapnikov, G., and Walraven, J. Regimes of quantum degeneracy in trapped 1d gases. Physical Review Letters 85, 3745 (2000).

[46] Berezinskii, V. Destruction of long-range order in one-dimensional and twodimensional systems possessing a continuous symmetry group. ii. quantum systems. Soviet Journal of Experimental and Theoretical Physics 34, 610 (1972).

[47] Kosterlitz, J. M. and Thouless, D. J. Ordering, metastability and phase transitions in two-dimensional systems. Journal of Physics C: Solid State Physics 6, 1181 (1973).

[48] Nitsche, W. H. et al. Algebraic order and the berezinskii-kosterlitz-thouless transition in an exciton-polariton gas. Physical Review B 90, 205430 (2014). 
[49] Kaitouni, R. I. et al. Engineering the spatial confinement of exciton polaritons in semiconductors. Physical Review B 74, 155311 (2006).

[50] Boiko, D. L. Towards R-space Bose-Einstein condensation ofphotonic crystal exciton polaritons. PIERS Online 4, 831-837 (2008).

[51] Winkler, K. et al. A polariton condensate in a photonic crystal potential landscape. New Journal of Physics 17, 023001 (2015).

[52] Tanese, D. et al. Fractal Energy Spectrum of a Polariton Gas in a Fibonacci Quasiperiodic Potential. Physical Review Letters 112, 146404 (2014).

[53] Krizhanovskii, D. et al. Effect of polariton-polariton interactions on the excitation spectrum of a nonequilibrium condensate in a periodic potential. Physical Review B 87, 155423 (2013).

[54] Tanese, D. et al. Polariton condensation in solitonic gap states in a one-dimensional periodic potential. Nature communications 4, 1749 (2013).

[55] Cerda-Méndez, E. et al. Exciton-polariton gap solitons in two-dimensional lattices. Physical Review Letters 111, 146401 (2013).

[56] Winkler, K. et al. Collective state transitions of exciton-polaritons loaded into a periodic potential. arXiv preprint arXiv:1509.08820 (2015).

[57] Carusotto, I. and Ciuti, C. Quantum fluids of light. Rev. Mod. Phys. 85, 299-366 (2013).

[58] Wouters, M., Liew, T. C. H., and Savona, V. Energy relaxation in one-dimensional polariton condensates. Physical Review B 82, 245315 (2010).

[59] Penckwitt, A. A., Ballagh, R. J., and Gardiner, C. W. Nucleation, growth, and stabilization of Bose-Einstein condensate vortex lattices. Physical Review Letters 89, 260402 (2002).

[60] Savenko, I. G., Liew, T. C. H., and Shelykh, I. A. Stochastic Gross-Pitaevskii Equation for the Dynamical Thermalization of Bose-Einstein Condensates. Physical Review Letters 110, 127402 (2013).

[61] Wouters, M. and Carusotto, I. Excitations in a nonequilibrium Bose-Einstein condensate of exciton polaritons. Physical Review Letters 99, 140402 (2007).

[62] Roumpos, G., Nitsche, W. H., Höfling, S., Forchel, A., and Yamamoto, Y. Gaininduced trapping of microcavity exciton polariton condensates. Phys. Rev. Lett. 104, 126403 (2010).

[63] Ostrovskaya, E. A., Abdullaev, J., Desyatnikov, A. S., Fraser, M. D., and Kivshar, Y. S. Dissipative solitons and vortices in polariton Bose-Einstein condensates. Physical Review A 86, 013636 (2012).

[64] Ge, L., Nersisyan, A., Oztop, B., and Tureci, H. E. Pattern formation and strong nonlinear interactions in exciton-polariton condensates. arXiv preprint arXiv:1311.4847 (2013).

[65] Keeling, J. and Berloff, N. G. Spontaneous rotating vortex lattices in a pumped decaying condensate. Physical Review Letters 100, 250401 (2008). 
[66] Tosi, G. et al. Sculpting oscillators with light within a nonlinear quantum fluid. Nature Physics 8, 190-194 (2012).

[67] Cristofolini, P. et al. Optical superfluid phase transitions and trapping of polariton condensates. Physical Review Letters 110, 186403 (2013).

[68] Askitopoulos, A. et al. Polariton condensation in an optically induced two-dimensional potential. Physical Review B 88, 041308 (2013).

[69] Dall, R. et al. Creation of orbital angular momentum states with chiral polaritonic lenses. Physical Review Letters 113, 200404 (2014).

[70] Borgh, M. O., Franchetti, G., Keeling, J., and Berloff, N. G. Robustness and observability of rotating vortex lattices in an exciton-polariton condensate. Physical Review B 86, 035307 (2012).

[71] Trallero-Giner, C. et al. Excited states of exciton-polariton condensates in $2 \mathrm{~d}$ and $1 \mathrm{~d}$ harmonic traps. Physical Review B 89, 205317 (2014).

[72] Balili, R., Snoke, D., Pfeiffer, L., and West, K. Actively tuned and spatially trapped polaritons. Applied Physics Letters 88, 031110 (2006).

[73] Vörös, Z., Snoke, D., Pfeiffer, L., and West, K. Trapping excitons in a twodimensional in-plane harmonic potential: experimental evidence for equilibration of indirect excitons. Physical Review Letters 97, 016803 (2006).

[74] Negoita, V., Snoke, D., and Eberl, K. Harmonic-potential traps for indirect excitons in coupled quantum wells. Physical Review B 60, 2661 (1999).

[75] de Lima Jr, M., van der Poel, M., Santos, P., and Hvam, J. M. Phonon-induced polariton superlattices. Physical Review Letters 97, 045501 (2006).

[76] Hofstetter, D., Maisenholder, B., and Zappe, H. P. Quantum-well intermixing for fabrication of lasers and photonic integrated circuits. IEEE journal of Selected Topics in Quantum Electronics 4, 794-802 (1998).

[77] Lu, E. H. Semiconductor quantum wells intermixing. Gordon \& Breach Science Publishers, (2000).

[78] Tan, H. H., Williams, J. S., Jagadish, C., Burke, P. T., and Gal, M. Large energy shifts in GaAs-AlGaAs quantum wells by proton irradiation-induced intermixing. Applied Physics Letters 68, 2401-2403 (1996).

[79] Zrenner, A. et al. Indirect excitons in coupled quantum well structures. Surface science 263, 496-501 (1992).

[80] Fraser, M., Tan, H., and Jagadish, C. Selective confinement of macroscopic longlifetime exciton and trion populations. Physical Review B 84, 245318 (2011).

[81] Gärtner, A., Prechtel, L., Schuh, D., Holleitner, A., and Kotthaus, J. Micropatterned electrostatic traps for indirect excitons in coupled GaAs quantum wells. Physical Review B 76, 085304 (2007).

[82] Butov, L., Lai, C., Ivanov, A., Gossard, A., and Chemla, D. Towards bose-einstein condensation of excitons in potential traps. Nature 417, 47-52 (2002). 
[83] Fisher, T. et al. Electro-optic tuning of vacuum Rabi coupling in semiconductor quantum microcavity structures. Solid-State Electronics 40, 493-496 (1996).

[84] Gessler, J. et al. Electro optical tuning of Tamm-plasmon exciton-polaritons. Applied Physics Letters 105, 181107 (2014).

[85] Brodbeck, S. et al. Room temperature polariton light emitting diode with integrated tunnel junction. Optics Express 21, 31098-31104 (2013).

[86] Tsotsis, P. et al. Tuning the Energy of a Polariton Condensate via Bias-Controlled Rabi Splitting. Physical Review Applied 2, 014002 (2014).

[87] Brodbeck, S. et al. Impact of lateral carrier confinement on electro-optical tuning properties of polariton condensates. Applied Physics Letters 107, 041108 (2015).

[88] Hammack, A. et al. Excitons in electrostatic traps. Journal of Applied Physics 99, 066104 (2006).

[89] Na, N. and Yamamoto, Y. Massive parallel generation of indistinguishable single photons via the polaritonic superfluid to mott-insulator quantum phase transition. New Journal of Physics 12, 123001 (2010).

[90] Wertz, E. et al. Spontaneous formation and optical manipulation of extended polariton condensates. Nature Physics 6, 860-864 (2010).

[91] Cristofolini, P. et al. Optical superfluid phase transitions and trapping of polariton condensates. Physical Review Letters 110, 186403 (2013).

[92] Cristofolini, P. et al. Geometrically locked vortex lattices in semiconductor quantum fluids. Nature Communications 3, 1243 (2012).

[93] Schmutzler, J. et al. All-optical flow control of a polariton condensate using nonresonant excitation. Physical Review B 91, 195308 (2014).

[94] Sun, Y. et al. Polaritons are not weakly interacting: Direct measurement of the polariton-polariton interaction strength. arXiv:1508.06698 (2015).

[95] Michler, P. Single Semiconductor Quantum Dots. Springer, (2009).

[96] Miller, R. et al. Trapped atoms in cavity QED: coupling quantized light and matter. Journal of Physics B: Atomic, Molecular and Optical Physics 38, S551 (2005).

[97] Reithmaier, J. P. et al. Strong coupling in a single quantum dot-semiconductor microcavity system. Nature 432, 197-200 (2004).

[98] Yoshie, T. et al. Vacuum Rabi splitting with a single quantum dot in a photonic crystal nanocavity. Nature 432, 200-203 (2004).

[99] Peter, E. et al. Exciton-photon strong-coupling regime for a single quantum dot embedded in a microcavity. Physical Review Letters 95, 067401 (2005).

[100] Press, D. et al. Photon antibunching from a single quantum-dot-microcavity system in the strong coupling regime. Physical Review Letters 98, 117402 (2007).

[101] Hennessy, K. et al. Quantum nature of a strongly coupled single quantum dot-cavity system. Nature 445, 896-899 (2007). 
[102] Kasprzak, J. et al. Up on the jaynes-cummings ladder of a quantum-dot/microcavity system. Nature materials 9, 304-308 (2010).

[103] Faraon, A. et al. Coherent generation of non-classical light on a chip via photoninduced tunnelling and blockade. Nature Physics 4, 859-863 (2008).

[104] Kistner, C. et al. Strong coupling in a quantum dot micropillar system under electrical current injection. Applied Physics Letters 96, 221102 (2010).

[105] Kistner, C. et al. Demonstration of strong coupling via electro-optical tuning in highquality qd-micropillar systems. Optics Express 16, 15006-15012 (2008).

[106] Nomura, M., Kumagai, N., Iwamoto, S., Ota, Y., and Arakawa, Y. Laser oscillation in a strongly coupled single-quantum-dot-nanocavity system. Nature Physics 6, 279-283 (2010).

[107] Schneider, C. et al. Single site-controlled $\operatorname{In}(\mathrm{Ga}) \mathrm{As} / \mathrm{GaAs}$ quantum dots: growth, properties and device integration. Nanotechnology 20, 434012 (2009).

[108] Schmidt, O. G. Lateral Alignment of Epitaxial Quantum Dots. Springer-Verlag Berlin Heidelberg, (2009).

[109] Mereni, L. O., Dimastrodonato, V., Young, R. J., and Pelucchi, E. A site-controlled quantum dot system offering both high uniformity and spectral purity. Applied Physics Letters 94, 223121 (2009).

[110] Kulakovskii, V. et al. Bose-Einstein condensation of exciton polaritons in high-Q planar microcavities with GaAs quantum wells. JETP letters 92, 595-599 (2010).

[111] Zajac, J. M. and Langbein, W. Structure and zero-dimensional polariton spectrum of natural defects in GaAs/AlAs microcavities. Physical Review B 86 (2012).

[112] Ding, F., Stoeferle, T., Mai, L. J., Knoll, A., and Mahrt, R. F. Vertical microcavities with high Q and strong lateral mode confinement. Physical Review B 87 (2013).

[113] Verger, A., Ciuti, C., and Carusotto, I. Polariton quantum blockade in a photonic dot. Physical Review B 73, 193306 (2006).

[114] Kim, N. Y. et al. Exciton-polariton condensates near the Dirac point in a triangular lattice. New Journal of Physics 15, 035032 (2013).

[115] Kaliteevski, M. et al. Tamm plasmon-polaritons: Possible electromagnetic states at the interface of a metal and a dielectric Bragg mirror. Physical Review B 76, 165415 (2007).

[116] Sasin, M. E. et al. Tamm plasmon polaritons: Slow and spatially compact light. Applied Physics Letters 92, 251112 (2008).

[117] Symonds, C., Lemaître, A., Homeyer, E., Plenet, J., and Bellessa, J. Emission of Tamm plasmon/exciton polaritons. Applied Physics Letters 95, 151114 (2009).

[118] Gazzano, O. et al. Evidence for confined Tamm plasmon modes under metallic microdisks and application to the control of spontaneous optical emission. Physical Review Letters 107, 247402 (2011). 
[119] Kim, N. Y. et al. Gaas microcavity exciton-polaritons in a trap. physica status solidi (b) 245, 1076-1080 (2008).

[120] Kochereshko, V. P. et al. Phase diagrams of magnetopolariton gases. arXiv preprint arXiv:1309.6983 (2013).

[121] Moreau, E. et al. Single-mode solid-state single photon source based on isolated quantum dots in pillar microcavities. Applied Physics Letters 79, 2865-2867 (2001).

[122] Santori, C., Fattal, D., Vuckovic, J., Solomon, G. S., and Yamamoto, Y. Indistinguishable photons from a single-photon device. Nature 419, 594-597 (2002).

[123] Heindel, T. et al. Electrically driven quantum dot-micropillar single photon source with 34\% overall efficiency. Applied Physics Letters 96, 011107 (2010).

[124] Silva, A. et al. Quantum dot dipole orientation and excitation efficiency of micropillar modes. Optics Express 16, 19201-19207 (2008).

[125] Ulrich, S. et al. Photon statistics of semiconductor microcavity lasers. Physical Review Letters 98, 043906 (2007).

[126] Reitzenstein, S. and Forchel, A. Quantum dot micropillars. Journal of Physics DApplied Physics 43, 033001 (2010).

[127] Gutbrod, T. et al. Weak and strong coupling of photons and excitons in photonic dots. Physical Review B 57, 9950 (1998).

[128] Obert, M. et al. Nonlinear emission in II-VI pillar microcavities: Strong versus weak coupling. Applied Physics Letters 84, 1435-1437 (2004).

[129] Bajoni, D. et al. Polariton laser using single micropillar gaas-gaalas semiconductor cavities. Physical Review Letters 100, 047401 (2008).

[130] Schmutzler, J. et al. Influence of interactions with noncondensed particles on the coherence of a one-dimensional polariton condensate. Physical Review B 89, 115119 (2014).

[131] Fischer, J. et al. Spatial coherence properties of one dimensional exciton-polariton condensates. Physical Review Letters 113, 203902 (2014).

[132] Dasbach, G., Bayer, M., Schwab, M., and Forchel, A. Spatial photon trapping: tailoring the optical properties of semiconductor microcavities. Semiconductor science and technology 18, S339 (2003).

[133] Bayer, M. et al. Optical demonstration of a crystal band structure formation. Physical Review Letters 83, 5374 (1999).

[134] Jacqmin, T. et al. Direct observation of Dirac cones and a flatband in a honeycomb lattice for polaritons. Physical Review Letters 112, 116402 (2014).

[135] Nguyen, H. S. et al. Realization of a double-barrier resonant tunneling diode for cavity polaritons. Physical Review Letters 110, 236601 (2013).

[136] Azzini, S. et al. Ultra-low threshold polariton lasing in photonic crystal cavities. Applied Physics Letters 99, 111106 (2011). 
[137] Zhang, B. et al. Zero-dimensional polariton laser in a subwavelength grating-based vertical microcavity. Light: Science \& Applications 3, e135 (2014).

[138] Fischer, J. et al. Magneto-exciton-polariton condensation in a sub-wavelength high contrast grating based vertical microcavity. Applied Physics Letters 104, 091117 (2014).

[139] Huang, M. C., Zhou, Y., and Chang-Hasnain, C. J. A surface-emitting laser incorporating a high-index-contrast subwavelength grating. Nature Photonics 1, 119122 (2007).

[140] Zhang, B. et al. Coupling polariton quantum boxes in sub-wavelength grating microcavities. Applied Physics Letters 106, 051104 (2015).

[141] Besga, B. et al. Polariton boxes in a tunable fiber cavity. Physical Review Applied 3, 014008 (2015).

[142] Dufferwiel, S. et al. Strong exciton-photon coupling in open semiconductor microcavities. Applied Physics Letters 104, 192107 (2014).

[143] El Daif, O. et al. Polariton quantum boxes in semiconductor microcavities. Applied Physics Letters 88, 061105 (2006).

[144] Mai, H. et al. 3d nanoimprinted fabry-pérot filter arrays and methodologies for optical characterization. Applied Physics B 107, 755-764 (2012).

[145] Pires, D. et al. Nanoscale three-dimensional patterning of molecular resists by scanning probes. Science 328, 732-735 (2010).

[146] Paraïso, T. et al. Enhancement of microcavity polariton relaxation under confinement. Physical Review B 79, 045319 (2009).

[147] Ostrovskaya, E. A., Abdullaev, J., Fraser, M. D., Desyatnikov, A. S., and Kivshar, Y. S. Self-localization of polariton condensates in periodic potentials. Physical Review Letters 110, 170407 (2013).

[148] Nalitov, A., , Solnyshkov, D., and Malpuech, G. Polariton z topological insulator. Physical Review Letters 114, 116401 (2015).

[149] Bardyn, C.-E., Karzig, T., Refael, G., and Liew, T. C. Topological polaritons and excitons in garden variety systems. Physical Review B 91, 161413(R) (2015).

[150] Gao, T. et al. Observation of non-Hermitian degeneracies in a chaotic exciton-polariton billiard. Nature 526, 554 (2015).

[151] Zhang, L. et al. Weak lasing in one-dimensional polariton superlattices. Proceedings of the National Academy of Sciences of the United States of America 112, 1516 (2015).

[152] Baboux, F. et al. Bosonic condensation and disorder-induced localization in a flat band. arXiv:1505.05652 (2015).

[153] Plumhof, J. D., Stöferle, T., Mai, L., Scherf, U., and Mahrt, R. F. Room-temperature bose-einstein condensation of cavity exciton-polaritons in a polymer. Nature Materials 13, 247-252 (2014). 
[154] Christopoulos, S. et al. Room-temperature polariton lasing in semiconductor microcavities. Physical Review Letters 98, 126405 (2007).

[155] Lu, T.-C. et al. Room temperature polariton lasing vs. photon lasing in a zno-based hybrid microcavity. Optics Express 20, 5530-5537 (2012).

[156] Liu, X. et al. Strong light-matter coupling in two-dimensional atomic crystals. Nature Photonics 9, 30-34 (2015). 